\title{
A Software Package to Simplify Tikhonov Regularization with Examples for Matrix-Based Inversion of SMPS and HTDMA Data
}

\author{
Markus D Petters ${ }^{1}$ \\ ${ }^{1}$ NC State University, Department of Marine, Earth, and Atmospheric Sciences, Raleigh, NC, 27695-8208 \\ Correspondence: Markus D Petters (mdpetter@ncsu.edu)
}

\begin{abstract}
Tikhonov regularization is a tool for reducing noise amplification during data inversion. This work introduces RegularizationTools.jl, a general-purpose software package to apply Tikhonov regularization to data. The package implements well-established numerical algorithms and is suitable for systems of up to 1000 equations. Included is an abstraction to systematically categorize specific inversion configurations and their associated hyperparameters. A generic interface translates arbitrary linear forward models defined by a computer function into the corresponding design matrix. This obviates the need to explicitly write out and discretize the Fredholm integral equation, thus facilitating fast prototyping of new regularization schemes associated with measurement techniques. Example applications include the inversion involving data from scanning mobility particle sizers (SMPS) and humidified tandem differential mobility analyzers (HTDMA). Inversion of SMPS size distributions reported in this work builds upon the freely-available software DifferentialMobilityAnalyzers.jl. The speed of inversion is improved by a factor of $\sim 200$, now requiring between 2 and 5 ms per SMPS scan when using 120 size bins. Previously reported occasional failure to converge to a valid solution is reduced by switching from the L-curve method to generalized cross-validation as the metric to search for the optimal regularization parameter. Higher-order inversions resulting in smooth, denoised reconstructions of size distributions are now included in DifferentialMobilityAnalyzers.jl. This work also demonstrates that an SMPS-style matrix-based inversion can be applied to find the growth factor frequency distribution from raw HTDMA data, while also accounting for multiply-charged particles. The outcome of the aerosol-related inversion methods is showcased by inverting multi-week SMPS and HTDMA datasets from ground-based observations, including SMPS data obtained at Bodega Bay Marine Laboratory during the Calwater 2/ACAPEX campaign, and co-located SMPS and HTDMA data collected at the U.S. Department of Energy observatory located at the Southern Great Plains site in Oklahoma, U.S.A. Results show that the proposed approaches are suitable for unsupervised, nonparametric inversion of large-scale datasets as well as inversion in real-time during data acquisition on low-cost reduced-instruction-set architectures used in single-board computers. The included software implementation of Tikhonov regularization is freely-available, general, and domain-independent, and thus can be applied to many other inverse problems arising in atmospheric measurement techniques and beyond.
\end{abstract}

\section{Introduction}

Atmospheric aerosol play an important role in shaping the microphysics of clouds and the Earth's climate (Farmer et al., 2015;

Kreidenweis et al., 2019). To predict the impact of aerosol on the Earth system, the distributions of particle size, chemical 
composition, hygroscopicity, and morphology must be known. Together these contribute to the mixing state of the aerosol. Accurate measurements of these distributions are critical for formulating models that link aerosol, cloud, and climate properties (Riemer et al., 2019).

Differential mobility analyzers (DMAs) select particles as a function of their size, charge, and an applied voltage. DMAs and tandem DMAs are widely used to measure the distributions of size and distributions of aerosol physicochemical properties (Park et al., 2008). For examples, a single DMA can be used to measure the aerosol size distribution by scanning voltage (Wang and Flagan, 1990). Humidified tandem DMAs (HTDMAs) can be used to measure the growth factor or hygroscopicity frequency distribution (Gysel et al., 2009). DMA-particle mass analyzer measurements can be used to resolve particle density distributions (Rawat et al., 2016; Sipkens et al., 2020). Tandem DMAs are important because they are one of only a handful techniques that can specifically characterize aspects of the aerosol mixing state (Riemer et al., 2019). Unfortunately, particles carrying multiple charges and different sizes transmit through the DMA at a single voltage, which creates artifacts in the raw instrument response that must be removed during post-processing of the data.

Humidified tandem DMAs select a single particle mobility diameter, pass this quasi-monodisperse aerosol through a humidification system, and then measure the humidified mobility response function using a second DMA operated in stepping or scanning mode (Rader and McMurry, 1986; Suda and Petters, 2013; Dawson et al., 2016). The humidified mobility response function is influenced by the particle size distribution, aerosol charge distribution, and growth factor frequency distribution function of the upstream aerosol. Gysel et al. (2009) show that the inversion from the humidified mobility response function to the growth factor frequency distribution is an ill-posed problem.

The inverse solution of ill-posed problems is characterized by strong sensitivity to noise superimposed on the data. Regularization methods are needed to relate an observed instrument response to the underlying physical property of the system under investigation. A common inverse method is $L_{2}$-regularization, developed independently by Phillips (1962), Twomey (1963), and Tikhonov (1963). Some examples of $L_{2}$-regularization involving atmospheric measurement techniques include inversion to find aerosol microphysical properties from measurements of optical properties (Dubovik and King, 2000; Müller et al., 2019), retrieve trace gas concentrations from remote sensors (Borsdorff et al., 2014), or estimate fluxes from a combination of measurements and atmospheric transport models (Krakauer et al., 2004). Application of $L_{2}$-regularization for problems involving DMAs include the reconstruction of the particle size distribution downstream of a single DMA (Wolfenbarger and Seinfeld, 1990; Kandlikar and Ramachandran, 1999; Talukdar and Swihart, 2003; Petters, 2018) and inversion to find size-mass distributions from coupled DMA-particle mass analyzer measurements (Rawat et al., 2016; Sipkens et al., 2020).

To date, $L_{2}$-regularization has not been applied to the inversion of HTDMA data. However, multiple other approaches have been used to estimate the growth factor frequency distribution from the humidified mobility response function. Stolzenburg and McMurry (1988) introduce the TDMAfit method. TDMAfit assumes a multi-mode normally-distributed hygroscopic growth factor frequency distribution. Parameters of the growth factor frequency distribution are varied such that the error between the modeled and observed humidified mobility response-functions are minimized. Cubison et al. (2005) apply the optimal estimation method (OEM) to derive the growth factor frequency distribution. This method uses an estimate of the covariance matrix, the measurements, and the forward model to retrieve the growth factor frequency distribution. The advantage of the 
optimal estimation method over TDMAfit is that it is nonparametric, i.e., it makes no prior assumption about the shape of the growth factor frequency distribution. However, the method sometimes produces oscillatory and negative solutions. Gysel et al. (2009) introduce TDMAinv, a piecewise linear version of TDMAfit. The piecewise method is also nonparametric. Constrained minimization is applied to find the growth factor frequency distribution; this avoids the negative solutions encountered in the optimal estimation method. Gysel et al. (2009) briefly discuss the role of multiple charges in the inversion and state that "the measured humidified mobility response function is a superposition of contributions from different dry sizes [...] and appropriate data inversion is hardly possible. Unfortunately an SMPS-style multicharge correction cannot be applied because the relative contributions from singly and multiply charged particles to every data point of the MDF cannot be distinguished." (In the direct quote, SMPS denotes scanning mobility particle sizer (Wang and Flagan, 1990) and MDF denotes mobility distribution function). Nevertheless, Shen et al. (2021) compute the contribution of multiply charged particles to the humidified mobility response function assuming that the larger multiply charged particles express the mean growth factor. However, they state that the correction of growth factor frequency distribution for multiply charged particles "is too complicated" (Shen et al., 2021) due to the need for multidimensional integration. Finally, Oxford et al. (2020) introduced a forward model named TAO that corrects for the contribution of multiply charged particle to the signal when interpreting volatility tandem DMA measurement.

This work revisits the challenge to perform an SMPS-style inversion of the humidified mobility distribution to retrieve the growth factor frequency distribution while also accounting for multiply charged particles. $L_{2}$-regularization is used to find the inverse. The remainder of the work is structured as follows: Section 2 describes the theory of $L_{2}$-regularization and the numerical solution of the equations. The software package RegularizationTools.jl is introduced, which is a general domainindependent implementation of $L_{2}$-regularization. Forward models for transfer through the single DMA and tandem DMA are formulated using the formalism developed in Petters (2018) and cast into matrix form using abstractions introduced in RegularizationTools.jl. Section 3 uses synthetic data to demonstrate that $L_{2}$-regularization can be used to invert the humidified mobility distribution function to find the growth factor frequency distribution. Section 4 uses real-world data to showcase improvements for size distribution inversion and the newly introduced tandem DMA inversion that were added to the freelyavailable software package DifferentialMobilityAnalyzers.jl (Petters, 2018). Finally, Section 5 summarizes the improvements, advantages, and limitations of the methodologies introduced in this work.

\section{Theory}

This work uses the following linear algebra notation. Capital bold-roman letters denote matrices, lower case roman letters denote vectors and italic symbols denote scalars. $\mathbf{A}^{\mathrm{T}}$ denotes the matrix transpose, and $\mathbf{A}^{+}$the matrix pseudo-inverse. 


\section{$2.1 \quad L_{2}$ Regularization}

\subsubsection{Theory}

The formalisms closely follow the description in Hansen (2000). Consider a system of equations

$\mathrm{b}=\mathbf{A x}+\epsilon$

where $\mathrm{b}$ is the measured response, $\mathbf{A}$ is the design matrix, $\mathrm{x}$ is the true quantity of interest, and $\epsilon$ is the random error. The regular least-squares solution computed using the pseudo inverse via $\mathrm{x}=\mathbf{A}^{+} \mathrm{b}$ is often dominated by contributions from the error and the thus-obtained estimate for $\mathrm{x}$ is useless. Regularization addresses this issue by solving the minimization problem

$\mathrm{x}_{\lambda}=\arg \min \left\{\|\mathbf{A} \mathrm{x}-\mathrm{b}\|_{2}^{2}+\lambda^{2}\left\|\mathbf{L}\left(\mathrm{x}-\mathrm{x}_{0}\right)\right\|_{2}^{2}\right\}$

where $\mathrm{x}_{\lambda}$ is the regularized estimate of $\mathrm{x},\|\cdot\|_{2}$ is the Euclidean norm, $\mathbf{L}$ is a filter matrix, $\lambda$ is the regularization parameter, and $\mathrm{x}_{0}$ is a vector of an a-priori estimate of the solution. The a-priori estimate can be taken to be $\mathrm{x}_{0}=0$ if no $a$-priori information is known. The filter matrix is often taken to be the identity matrix $\mathbf{I}$ or a derivative operator. Common choices are the first and second derivative operator defined as the bidiagonal $(-1,1)$ and the upper tridiagonal $(1,-2,1)$ matrix, respectively. For $\lambda=0$, the solution is equivalent to $\mathrm{x}_{\lambda}=\mathbf{A}^{+} \mathrm{b}$. In the limit $\lim _{\lambda \rightarrow \infty} \mathrm{x}_{\lambda}=\mathrm{x}_{0}$. Thus the regularization parameter "interpolates" between the noisy ordinary least squares solution and the a-priori estimate $\mathrm{x}_{0}$.

The analytical solution for Eq. (2) is the regularized normal equation

$\mathrm{x}_{\lambda}=\left(\mathbf{A}^{\mathrm{T}} \mathbf{A}+\lambda^{2} \mathbf{L}^{\mathrm{T}} \mathbf{L}\right)^{-1}\left(\mathbf{A}^{\mathrm{T}} \mathrm{b}+\lambda^{2} \mathbf{L}^{\mathrm{T}} \mathbf{L} \mathrm{x}_{0}\right)$

which is derived by taking the derivative of the right hand side of Eq. (2), setting it to zero, and solving for x. Equation (3) is in standard form if $\mathbf{L}=\mathbf{I}$. The optimal regularization parameter can be obtained using a variety of techniques, including the $\mathrm{L}$ curve method (Hansen, 2000) and generalized cross-validation (GCV, Golub et al., 1979). The L-curve methods involves a plot of $\log \|\mathbf{A x}-\mathrm{b}\|_{2}^{2}$ vs. $\log \left\|\mathbf{L}\left(\mathrm{x}-\mathrm{x}_{0}\right)\right\|_{2}^{2}$. The optimal $\lambda$ occurs at the corner of the L-curve, which can be found algorithmically. The generalized cross-validation estimator is

$110 V(\lambda)=\frac{n\left\|\left(\mathbf{I}-\mathbf{A}_{\lambda}\right) \mathrm{b}\right\|_{2}^{2}}{\operatorname{tr}\left(\mathbf{I}-\mathbf{A}_{\lambda}\right)^{2}}$

where $\mathbf{A}_{\lambda}=\mathbf{A}\left(\mathbf{A A}^{T}-\lambda^{2} \mathbf{I}\right)^{-1} \mathbf{A}^{T}$ is the influence matrix, $t r$ is the matrix trace, and $n$ is the size of b. The optimal $\lambda_{o p t}$ coincides with the global minimum of $V(\lambda)$. Equation (4) requires that the system is in standard form. For systems in nonstandard form, conversion to standard form is required before computing $V(\lambda)$. In many cases $\lambda_{o p t}$ found by the L-curve and generalized cross-validation are similar, and the retrieved solutions $\mathrm{x}_{\lambda}$ are nearly indistinguishable. Differences between these two estimates are related to the computational speed-to-converge and robustness, i.e., that the system converges to the optimal solution. 


\subsubsection{Algorithms}

Equation (3) can be solved straightforwardly using any software that supports linear algebra operations. This brute force approach, however, is slow. Efficient algorithms to solve Eqs. (3) and (4) have been developed. The algorithms used here are briefly described. The regularized normal equations are transformed to standard form using the generalized singular value decomposition of A and $\mathbf{L}$ as derived by Eldén (1982) and summarized by Hansen (1998). Equation (3) is solved using Cholesky factorization when possible since it is the computationally fastest approach (Lira et al., 2016). If Cholesky factorization fails, one of the fallback solvers selected by the linear algebra package of the programming language is used. Equation (4) is solved using the singular value decomposition of $\mathbf{A}$ and the iterative algorithm described in Bates et al. (1986). The optimal $\lambda_{\text {opt }}$ for generalized cross-validation is found by minimizing $V(\lambda)$ on a bounded interval using Brent's method (Mogensen and Riseth, 2018). The optimal $\lambda_{\text {opt }}$ for the L-curve method is found by maximizing Eq. (18) in Hansen (2000) on a bounded interval using Brent's method.

\subsubsection{Classification of Methods}

The inverse problem can be solved using specific methods. Here, method refers to the content of the filter matrix $\mathbf{L}$, whether an a-priori estimate is used, and whether constraints are imposed on the solution. Methods are encoded through the following expression

$L_{k} x_{0} D_{\epsilon} B_{[l b, u b]}(a l g)$

where $L_{k}$ denotes the order of the filter matrix $\mathbf{L}, x_{0}$ denotes whether an a-priori estimate is used, $D_{\epsilon}$ denotes whether data-based constraints are used (explained further below), and $B_{[l b, u b]}$ denotes whether a lower bound (lb) or upper bound (ub) is imposed on the solution (explained further below). The argument ("alg") denotes constraints on the search algorithms, e.g., L_curve or gcv and/or the bounded interval over which $\lambda$ is varied. The expression is composable. For examples, the method $L_{2}$ denotes inversion using the second order derivative without an a-priori estimate, data-based constraints, and lower/upper bound constraints. The method $L_{0} B_{[0,1]}\left(\right.$ alg $\left.=L \_c u r v e, \lambda_{1}=0.01, \lambda_{2}=100\right)$ denotes inversion with $\mathbf{L}=\mathbf{I}$, imposing that all $\mathrm{x}_{\lambda} \in[0,1]$, the use of the $\mathrm{L}$-curve method, and $\lambda_{\text {opt }} \in[0.01,100]$. If alg is unspecified, defaults of $a l g=g c v, \lambda_{1}=0.001, \lambda_{2}=1000$ are implied. This approach of method encoding provides a convenient classification system to enumerate the set of available methods as well as to specify the method in a high-level application interface for software function calls. There are eight combinations by which to compose methods via Eq. (5); combined with the three most common filter matrices $L_{0}=\mathbf{I}, L_{1}=\operatorname{bidiagonal}(-1,1)$ and, $L_{2}=$ upper tridiagonal $(1,-2,1)$ this results in 24 unique methods.

Data-based constraints: Huckle and Sedlacek (2012) proposed a two-step data-based regularization where the filter matrix is modified according to

$\mathbf{L}=\mathbf{L}_{k} \mathbf{D}_{\hat{x}}^{-1}$ 
where $\mathbf{L}_{k}$ is one of the finite difference approximations of a derivative, $\mathbf{D}_{\hat{x}}=\operatorname{diag}\left(\left|\hat{x_{1}}\right|, \ldots\left|\hat{x_{n}}\right|\right), \hat{x}$ is the reconstruction of $x$ using $\mathbf{L}_{k}$, and $\left(\mathbf{D}_{\hat{x}}\right)_{i i}=\epsilon \forall\left|\hat{x}_{i}\right|<\epsilon$, with $\epsilon<<1$. The method $L_{1} D_{0.01}$ represents a filter matrix with a first-order derivative operator applied to Eq. (6) with $\epsilon=0.01$.

Lower/upper bound constraints: The retrieved $\mathrm{x}_{\lambda}$ from the regularized normal equation can have oscillatory and/or nonphysical solutions. An alternative approach is to treat Eq. (2) as a constrained minimization such that the solution is subject to the optional constraint $\mathrm{x}_{l b}<\mathrm{x}<\mathrm{x}_{u b}$. Here, the following procedure is implemented for the bounded search: first, the optimal $\lambda_{o p t}$ is found using the regularized normal equations. The thus-obtained solution $\mathrm{x}_{\lambda}$ is truncated at the upper and lower bounds and then passed as an initial condition to a least-squares numerical solver. The Ceres solver (Agarwal et al.) is used with the Dogleg method and QR solver as implemented in the freely-available LeastSquaresOptim.jl ${ }^{1}$ library. The upper and lower bounds are vectors of the same size asx.

\subsubsection{Software Implementation}

$L_{2}$-regularization, as described in the previous sections, is implemented in a freely-available software package RegularizationTools.jl that is written by the author and provided as a supplement to this work. The implementation is in the Julia programming language (Bezanson et al., 2017). The package has a similar name and some overlap with the package Regularization Tools by Hansen (2007). However, the packages differ in software architecture, programming language, and scope. RegularizationTools.jl provides a simple high-level interface to compute $\mathrm{x}_{\lambda}$ using a single function call, for example

$\mathrm{x} \lambda=\operatorname{invert}\left(\mathrm{A}, \mathrm{b}, \mathrm{L}_{\mathrm{k}} \mathrm{x}_{0} \mathrm{~B}\left(\mathrm{k}, \mathrm{x}_{0}, \mathrm{lb}, \mathrm{ub}\right) ; \mathrm{alg}=: \mathrm{L} \_\right.$curve $)$

where $\mathrm{A}$ is the design matrix, $\mathrm{b}$ is the observation vector, $\mathrm{L}_{\mathrm{k}} \mathrm{x}_{0} \mathrm{~B}$ is a parameterized algebraic data type that encodes the specific method. The hyper parameter $\mathrm{k}$ specifies the order, $\mathrm{x}_{0}$ specifies a vector of the initial guess, $\mathrm{lb}$ and ub specifies vectors of the lower and upper bounds. Other methods can be specified according to Eq. (5). Examples are provided in the documentation of the package.

\subsection{Computing the Design Matrix}

The design matrix can be obtained from a forward model

$\mathrm{y}=F(\mathrm{x}, \mathrm{c})$

where $\mathrm{y}$ is a vector representing the error-free observations, $\mathrm{x}$ is the vector of true inputs, $\mathrm{c}$ is a vector of controlling parameters, and $F$ is the linear forward model function that maps over $\mathrm{x}$ to compute $\mathrm{y}$ subject to the constraint of $\mathrm{c}$. The matrix of the linear transformation $\mathrm{y}=\mathbf{A x}$ is then given by

$\mathbf{A}=\left[F\left(\mathrm{e}_{1}\right) F\left(\mathrm{e}_{2}\right) \ldots F\left(\mathrm{e}_{n}\right)\right]$

\footnotetext{
${ }^{1}$ https://github.com/matthieugomez/LeastSquaresOptim.jl
} 
where $\mathrm{e}_{1}, \ldots, \mathrm{e}_{n}$ is the standard basis. RegularizationTools.jl also provides abstract generic interface that simplifies computation of the design matrix from arbitrary forward models of linear processes. Examples demonstrating how to use this generic interface are provided in the documentation of the package. The examples include the solution for transit through the tandem DMA described further below, the solution of the Fredholm integral equation of the first kind given by Baart (1982), the optical convolution that underlies size distribution retrieval from scattering and absorption properties (Müller et al., 2019), and the 2D Gaussian blur function encountered in image processing.

\subsection{Design Matrices For Differential Mobility Analyzers}

Differential mobility analyzers consist of two electrodes held at a constant- or time-varying electric potential. Cylindrical (Knutson and Whitby, 1975) and radial (Zhang et al., 1995; Russell et al., 1996) electrode geometries are the most common. Charged particles are deflected to an exit slit and measured by a suitable detector, usually a condensation particle counter. The fraction of particles carrying $k$ charges is described by a statistical distribution that is created by the charge conditioner used upstream of the DMA. The functions governing the transfer through bipolar charge conditioners, single DMAs, and tandem DMAs is well understood (Knutson and Whitby, 1975; Rader and McMurry, 1986; Reineking and Porstendörfer, 1986; Wang and Flagan, 1990; Stolzenburg and McMurry, 2008; Jiang et al., 2014). These functions are usually cast in the form of a Fredholm integral equation and discretized to find the inversion matrix.

Petters (2018) introduced a formal language to simplify this process. At the core of the language are size distributions encoded as a SizeDistribution composite data type. Composite data types combine multiple arrays into a single symbol for ease of use, facilitating faster experimental design and analysis. SizeDistribution consists of vectors of bin edges, bin midpoints, number concentration, log-normalized spectral density, and logarithmic bin widths. SizeDistributions are denoted in blackboard bold font (e.g., $n, r$, etc.). SizeDistributions are the building block of composable algebraic expressions that evaluate to transformed SizeDistributions. For examples, $\mathrm{n}_{1}+\mathrm{m}_{2}$ is the superposition of two size distributions, $f * \mathrm{~m}$ is the uniform scaling of the concentration fields by factor $f, T * * \mathrm{~m}$ is the elementwise scaling of the concentration fields by factor $T, \mathbf{A} * \mathrm{~m}$ is matrix multiplication of $\mathbf{A}$ and concentration fields of the size distribution, $f \cdot \mathbf{m}$ is the uniform scaling of the diameter field of the size distribution by factor $f$, and $T \cdot \mathrm{m}$ is the elementwise scaling of the diameter field by factor $T$. DMA geometry, dimensions, and configuration are abstracted into composite types $\Lambda$ (configuration comprising flow rates, power supply polarity, and thermodynamic state) and $\delta$ (DMA domain defined by a mobility/size grid). Each DMA is fully described by a pair $\Lambda, \delta$. Subscripts and superscripts are used to distinguish between different configurations in chained DMA setups, e.g. $\delta_{1}$ and $\delta_{2}$ denoting the first and second DMA, respectively. Application of size distribution expressions to transfer functions constructs a concise model of the transmitted DMA mobility distribution, denoted as the DMA response function. Implementation of the language is distributed through a freely-available and independently documented package DifferentialMobilityAnalyzers.jl, written in the Julia language.

Petters (2018) shows that the convolution matrix for passage through a single DMA is given by the expression

$$
\mathbf{A}=\left(\operatorname{hcat}\left(\operatorname{map}\left(z^{s} \rightarrow \sum\left(k \rightarrow T_{s i z e}^{\Lambda, \delta}\left(k, z^{s}\right), m\right), Z\right) \ldots\right)\right)^{T}
$$


where $\sum(f, m)$ evaluates the function $f(x)$ for $x=[1, \ldots, m]$ and sums the result, $m$ is the upper number of multiply charged particles, $Z$ is a vector of centroid mobilities scanned by the DMA, hcat() is a function that concatenates arrays, “...” is the Julia splatting operator, which combines multiple arguments passed to the function hcat() into a single variable, and $T_{\text {size }}^{\Lambda, \delta}\left(k, z^{s}\right)=$ $\Omega\left(Z, z_{k}^{s}\right) * * T_{c}\left(k, D_{p, 1}\right) * * T_{l}\left(D_{p, 1}\right)$ is a function that evaluates to a vector representing the fraction of particles carrying $k$ charges that exit $\mathrm{DMA}^{\Lambda, \delta}$ as a function of diameter, $\Omega$ is the diffusing DMA transfer function (Stolzenburg and McMurry, 2008), $T_{c}$ is the charge frequency distribution (Wiedensohler, 1988), and $T_{l}$ is the diameter-dependent transmission loss (Reineking and Porstendörfer, 1986). The size distribution after passage through the DMA is given by $\mathrm{r}=\mathbf{A m}+\epsilon$, where $\mathrm{r}$ is the response function, $\mathrm{m}$ is the true size distribution, and $\epsilon$ is a vector denoting the random error that may be superimposed as a result of measurement uncertainties.

The mobility distribution exiting DMA 2 in the humidified tandem DMA is evaluated using the expressions

$\mathbb{M}_{k}^{\delta_{1}}=\left(\frac{D_{1}\left(z^{s}\right)}{D_{k}\left(z^{s}\right)}\right) \cdot\left(g f_{k}\left(z^{s}, g f_{0}\right) \cdot\left(T_{s i z e}^{\Lambda, \delta}\left(k, z^{s}\right) * \mathbb{m}^{c n}\right)\right)$

$\mathrm{m}_{t}^{\delta_{2}}=\mathbf{O} *\left(\sum_{k=1}^{m} \mathbf{M}_{k}^{\delta_{1}}\right)$

In Eq. (11), $\mathrm{M}_{k}^{\delta_{1}}$ evaluates to the size distribution of particles that exit the $\mathrm{DMA}^{\Lambda, \delta}$ at the nominal setpoint-diameter defined by mobility $z^{s}$ (or z-star) in DMA 1 and particle charge $k$. Subscripts are used to differentiate DMA 1 and 2 which possibly have different geometries, flow rates, and grids, e.g. $\Lambda_{1}, \Lambda_{2}$ and $\delta_{1}, \delta_{2} . D_{k}\left(z^{s}\right)$ is the mobility diameter selected by the DMA if the particle carries $k$ charges, $g f_{0}=D_{\text {wet }} / D_{d r y}$ is the true diameter growth factor, $D_{d r y}$ is the selected diameter by DMA $1, D_{\text {wet }}$ is the diameter after the humidifier, $g f_{k}\left(z^{s}, g f_{0}\right)$ is the effective growth factor for particles carrying $k$ charges, $T_{\text {size }}^{\Lambda, \delta}\left(k, z^{s}\right)$ is as in Eq. (10), and $\mathrm{m}^{c n}$ is the size distribution upstream of DMA 1. In Eq. (12), $\mathrm{m}_{t}^{\delta_{2}}$ is the total humidified mobility distribution exiting DMA 2, $m$ is upper number of charges on the multiply charged particles, $Z$ is a vector of centroid mobilities scanned by DMA 2 , and $\mathbf{O}=\left(\operatorname{hcat}\left(\operatorname{map}\left(z^{s} \rightarrow \sum\left(\mathrm{k} \rightarrow \Omega^{\Lambda_{2}, \delta_{2}}\left(Z, z_{k}^{s}\right) . * T_{l}^{\Lambda_{2}, \delta_{2}}\left(D_{p, 1}\right), 1\right), Z\right) \ldots\right)\right)^{T}$ is the convolution matrix for transport through DMA 2. Equations (11) and (12) are identical to those given in Petters (2018), except for the $g f_{k}\left(z^{s}, g f_{0}\right)$ function, which was then assumed to be a constant. This is incorrect. Particles carrying more than a single charge alias at a smaller particle size (Gysel et al., 2009; Shen et al., 2021). The effect is due to the size dependence of the slip-flow correction factor. The function must be solved iteratively and is now included in the DifferentialMobilityAnalyzers.jl package. Equation (11), and specifically $g f_{k}\left(z^{s}, g f_{0}\right)$, assumes that $g f_{0}$ applies to all particle sizes.

If the aerosol is externally mixed, the humidified distribution function is given by

$\mathrm{m}_{t}^{\delta_{2}}=\int_{0}^{\infty} P_{g f}\left[\mathbf{O} *\left(\sum_{k=1}^{m} \mathbf{M}_{k}^{\delta_{1}}\right)\right] d g f_{0}$ 

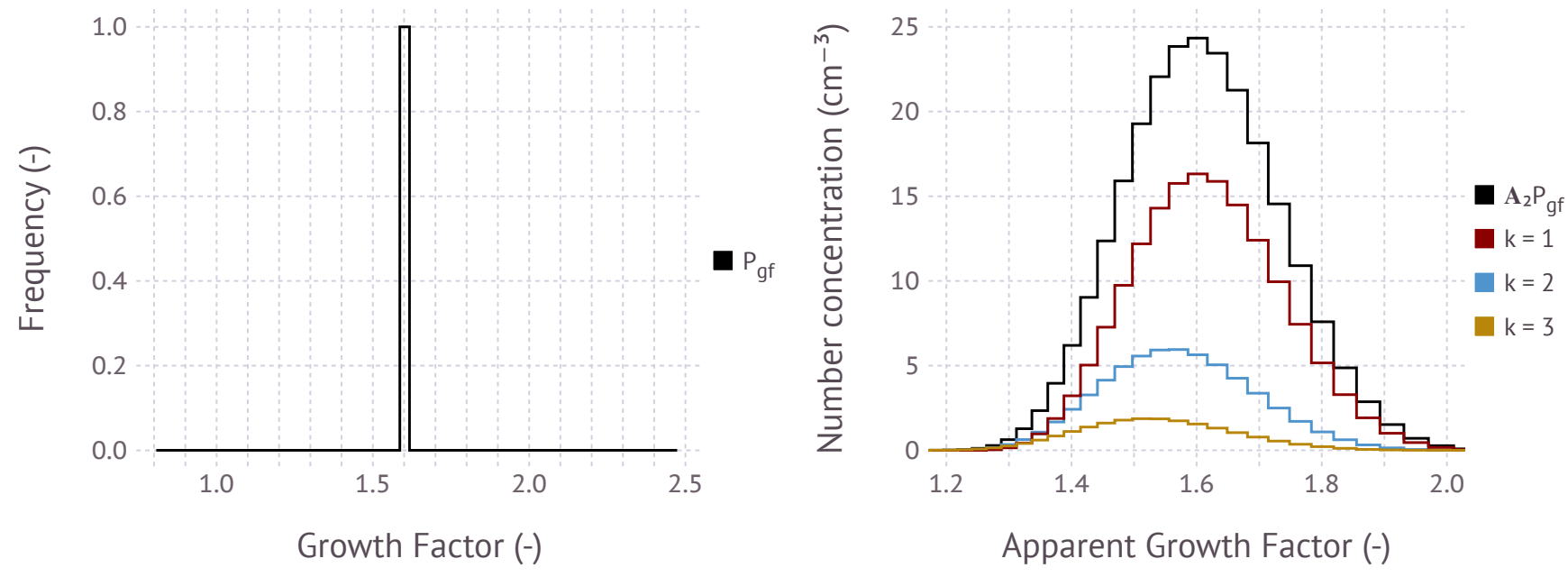

Figure 1. Left: Input growth factor frequency distribution assuming that all particles have a single growth factor $\sim 1.6$. Right: Modeled apparent mobility distribution function calculated using Eq. (15) and partial distributions for individual charges $k=+1,+2,+3$ computed via $\mathbf{O} * \mathrm{M}_{k}^{\delta_{1}}$. The example is free of measurement error, i.e., $\epsilon=0$. The black trace is observed.

where $P_{g f}$ is the growth factor frequency distribution. $\mathrm{m}_{t}^{\delta_{2}}$ in Eq. (13) is the forward model through the tandem DMA. Using the notation in section 2.2 ,

$F(\mathrm{x}, \mathrm{c})=\int_{0}^{\infty} P_{g f}\left[\mathbf{O} *\left(\sum_{k=1}^{m} \mathrm{M}_{k}^{\delta_{1}}\right)\right] d g f_{0}$

where $\mathrm{x}$ is the true $P_{g f}$ and the vector c of constraining parameters comprises the DMA setup $\Lambda_{1}, \Lambda_{2}, \delta_{1}, \delta_{2}$ and upstream size distribution $\mathrm{m}^{c n}$. Computer code that creates a forward model for tandem DMAs has been added to the DifferentialMobiltyAnalyzers.jl package and is annotated in the documentation of the package. The forward model is used to cast Eq. (14) into matrix form such that the humidified mobility distribution function is given by

$\mathrm{m}_{t}^{\delta_{2}}=\mathbf{A}_{2} P_{g f}+\epsilon$

where the subscript 2 specifies transmission through DMA 2, the matrix $\mathbf{A}_{2}$ is understood to be computed for a specific input aerosol size distribution, and $\epsilon$ is a vector that denotes the random error that may be superimposed as a result of measurement uncertainties. The size of $\mathbf{A}_{2}$ is $n^{2}$, where $n$ is the number of bins in the growth factor frequency distribution.

Figure 1 shows an example application of Eq. (15) for an input growth factor frequency distribution where all particles are assumed to have the same growth factor $\sim 1.6$. The frequency distribution is evaluated along a discrete growth factor grid with 60 bins. The assumed input size distribution is bimodal with mode diameters of 60 and $140 \mathrm{~nm}$, geometric standard deviations of 1.4 and 1.6, and number concentrations of 1300 and $2000 \mathrm{~cm}^{-3}$ in modes 1 and 2, respectively. The assumed sheath-tosample flow ratios are 5:1 in both DMAs. The product $\mathbf{A}_{2} P_{g f}$ is the raw response that would be measured by a condensation 

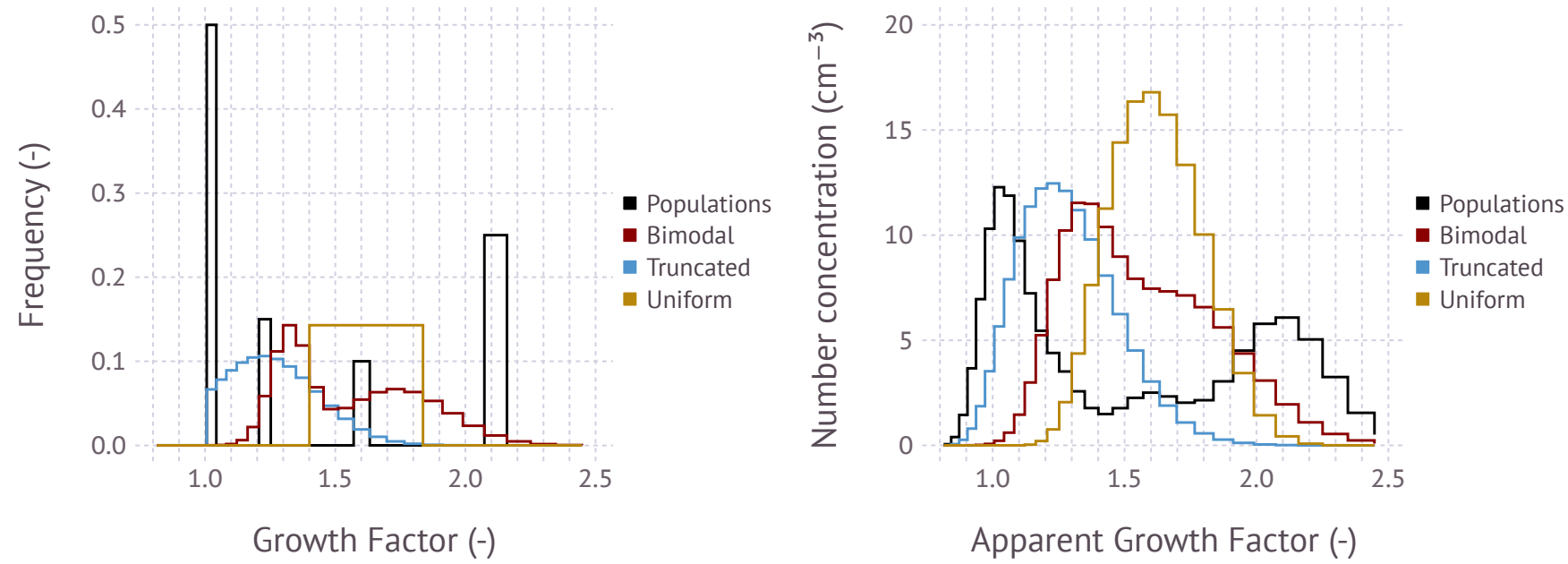

Figure 2. Left: Illustrative input growth factor frequency distributions. Right: Corresponding modeled apparent mobility distribution function calculated using Eq. (15). The example is free of measurement error, i.e. $\epsilon=0$.

particle counter at the exit of the instrument. Contribution of $+1,+2$, and +3 charged particles to the total can be computed via $\mathbf{O} * \mathrm{M}_{k}^{\delta_{1}}$. Although the nominal growth factor is the same for all sizes, the apparent mode of the growth factor decreases with increasing particle charge (see also Gysel et al., 2009; Shen et al., 2021). Therefore the axis is denoted as the apparent growth factor. Summing the partial distributions results in $\mathbf{A}_{2} P_{g f}$, demonstrating that the matrix equation correctly maps $P_{g f}$ to the response, including multiply charged particles.

Figure 2 shows the relationship between four illustrative growth factor frequency distributions and the modeled apparent mobility distribution functions. The selected examples comprise a testbed to evaluate the feasibility of an SMPS-style matrixbased inversion to recover $P_{g f}$. The Populations example consists of an external mixture with compositions corresponding to four unique growth factors. The Bimodal example is the superposition of two Gaussian distributions with $70 \%$ of particles in the less hygroscopic mode. The Truncated example is a Gaussian distribution truncated at $g f=1.0$. The Uniform example is a uniform distribution over a fixed interval. All frequency distributions sum to unity, thus accounting for $100 \%$ of the particle population. The dry diameter and assumed input size distribution to compute the matrix $\mathbf{A}_{2}$ is the same as in Fig. 1. However, unlike in Fig. 1, the frequency distribution and matrix are evaluated along a coarser discrete growth factor grid with 30 bins. Note that the growth factor bin width is not constant, with wider bins at larger growth factors. This is due to the evaluation of the humidified size distribution along a geometrically stepped mobility grid, which is typical in scanning DMA setups. As will be shown next, 30-bin resolution is a suitable compromise between speed, accuracy, and resolution when computing the matrix-based inversion to infer $P_{g f}$ from noise-perturbed apparent growth factor frequency distributions. 

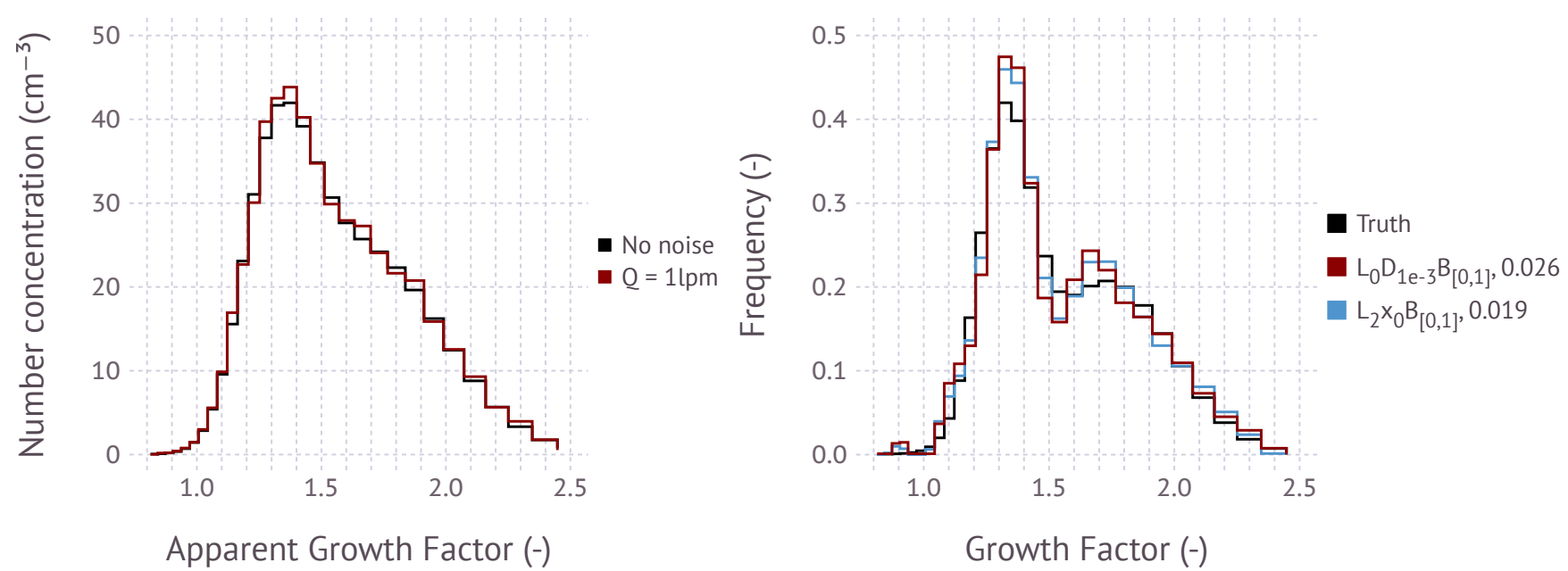

Figure 3. Left: Humidified apparent growth factor distribution function for the Bimodal example comprising superposition of two Gaussian distributions with $70 \%$ of particles in the less hygroscopic mode. The distributions are calculated $m_{t}^{\delta_{2}}=\mathbf{A}_{2} P_{g f}+\epsilon$. "No noise" corresponds to $\epsilon=0$. " $Q=1 \mathrm{lpm}$ " corresponds to simulated Poisson noise equivalent for a condensation particle counter measuring at a flow rate of 1 $\mathrm{L} \min ^{-1}$ and bin integration time of 2 seconds per bin. Right: Inverted size distribution using the $L_{0} D_{1 e-3} B_{[0,1]}$ and $L_{2} x_{0} B_{[0,1]}$ method. The a-priori estimate $\mathrm{x}_{0}$ is the normalized apparent growth factor distribution. Values in the legend $(0.026$ and 0.019$)$ correspond to the root mean square error between the true input (Truth) and the regularized solution.

\section{Matrix Inversion of the Humidified Mobility Distribution Function using Synthetic Data}

Simulated examples are used to test if Eq. (15) is invertible. Figure 3 shows an example simulation for the Bimodal growth factor distribution test case. The humidified apparent growth factor distributions are calculated using Eq. (15). The noisefree example corresponds to $\epsilon=0$ and represents the idealized measurement. Poisson counting statistics are simulated by converting concentration to the expected number of counts for a typical particle counter flow rate and bin integration time. Counts in each bin are computed by drawing a pseudo-random number from a Poisson distribution and converting the result back to concentration. Lower flow rates and shorter integration times increase the noise-perturbation of the apparent growth factor distribution. The apparent growth factor distribution is then inverted using the $L_{0} D_{1 e-3} B_{[0,1]}$ and $L_{2} x_{0} B_{[0,1]}$ method. Here $B_{[0,1]}$ is shorthand for setting all lower bounds equal to zero and all upper bounds equal to one. The a-priori estimate $\mathrm{x}_{0}$ is taken to be the normalized apparent growth factor distribution, where the normalization ensures that the sum over all bins is unity. Since the true noise-free input growth factor frequency distribution is known, the fidelity of the inversion can be evaluated by computing the root mean square error between the noise-free solution and the regularized solution. The figure shows that both inversion methods produce a root mean square error between 0.02 and 0.03 . These values are typical for the of reconstruction (see supporting information). Visual evaluation of the agreement between the reconstruction and the input suggest that either method is suitable for inversion. 

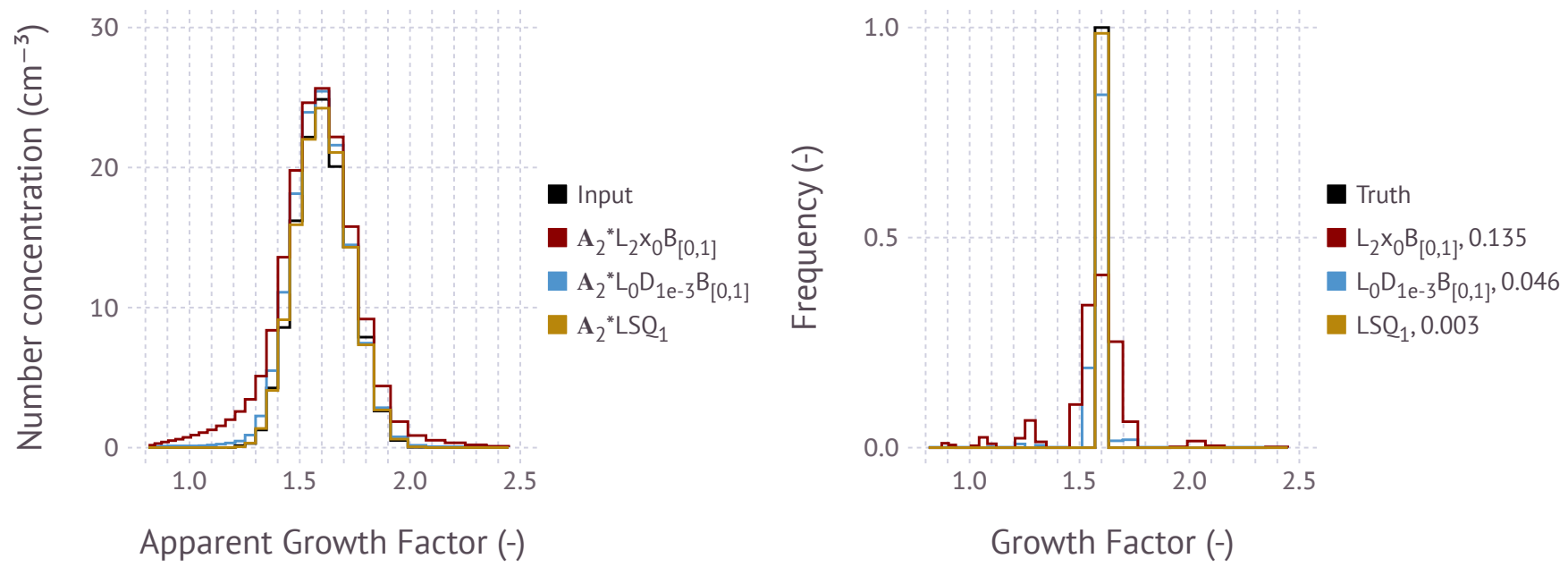

Figure 4. Left: Humidified apparent growth factor distribution function assuming that all particles have a single growth factor $\sim 1.6$. The black histogram corresponds to the input to the inversion, which is the noise-perturbed apparent growth factor distribution with simulated Poisson noise equivalent for a condensation particle counter measuring at a flow rate of $1 \mathrm{~L} \mathrm{~min}^{-1}$ and bin integration time of $2 \mathrm{~s}$ per bin. Color lines depict the predicted apparent growth factor distributions based on the corresponding inversion shown in the right panel. Right: Inverted growth factor frequency distribution from the noise-perturbed spectrum. The true growth factor frequency distribution (black line) is obscured behind the gold and blue lines and is as in Fig. 1, left panel. Colors correspond to the inverted size distribution using the $L_{0} D_{1 e-3} B_{[0,1]}$, $L_{2} x_{0} B_{[0,1]}$, and $L S Q_{1}$ methods. The a-priori estimate $\mathrm{x}_{0}$ is the normalized apparent growth factor distribution. Values in the legend $(0.135$, ...) correspond to the root mean square error between the true noise-free solution and the proposed solution.

Figure 4 is similar to Figure 3, showing an example simulation for an aerosol with uniform composition, i.e., all particles have the same growth factor. Although the $L_{2} x_{0} B_{[0,1]}$ approach correctly infers the most probable growth factor, the predicted distribution is incorrect. Multiple modes to the left and right of the main mode are observed. The $L_{2} x_{0}$ method produces an oscillatory solution with negative values (not shown). The small modes are the residual of this oscillatory solution that is truncated by the enforced $[0,1]$ bound and the inability of the least-squares solver to converge on a better solution. A large root mean square error of 0.135 results. In contrast, the data-constrained method $L_{0} D_{1 e-3} B_{[0,1]}$ leads to better reconstruction of the true input. The main advantage of the $L_{0} D_{1 e-3} B_{[0,1]}$ inversion method over $L_{2} x_{0} B_{[0,1]}$ is that it is better able to reconstruct inputs with sharp edges.

The total number of composable regularization methods according to Eq. (5) is 24. Half of these methods do not include lower and upper bounds and these are not suitable for tandem DMA inversion due to the negative and oscillatory solutions for narrow inputs. The remaining 12 methods have been systematically tested using Monte-Carlo analysis described in detail in the supporting information. Briefly, 60000 inversions were performed on synthetic data similar to the examples shown in Figs. 3 and 4 . The total number concentration, dry diameter, number of bins, and random seeds were varied and the root mean square error was evaluated for each simulation. Results compiled in Fig. S1 show that all of the methods perform equally well for the 
Bimodal, Uniform, and Truncated Normal examples shown in Fig. 3. Method $L_{0} D_{1 e-3} B_{[0,1]}$ outperforms the other methods for grids with 20 and 30 growth factor bins and test cases with either one (e.g., Fig. 4) or two discrete populations. However, even $L_{0} D_{1 e-3} B_{[0,1]}$ can lead to results similar to the example $L_{2} x_{0} B_{[0,1]}$ shown in Fig. 4 for some random seeds. Higher resolution grids generally lead to poor performance even for method $L_{0} D_{1 e-3} B_{[0,1]}$.

An alternative approach to fit single component data is to perform a nonlinear least squares fit to match the apparent growth factor distribution using the forward model while restricting the number of compositions to either one or two. This corresponds to a two- or four-parameter fit. Results from this procedure are either one or two growth factors and one or two fractions. The corresponding methods are denoted as $L S Q_{1}$ and $L S Q_{2}$, respectively. In the example shown in Fig. 4, $L S Q_{1}$ has the smallest root mean square error and is the best method to reconstruct the true growth factor. The $L S Q_{1}$ method is most suitable to infer the growth factor for laboratory measurements when it is known that the aerosol is internally mixed and only a single growth factor is expected.

Which method, however, should be selected when inverting real-world data and the number of components is unknown? Since the true solution is also unknown, the root mean square error between the truth and reconstruction is unavailable. It is, however, possible to compute the residual between the measured apparent growth factor distribution and the predicted apparent growth factor distribution from different reconstructions. A large residual can be used to flag truncated oscillatory solutions such as $L_{2} x_{0} B_{[0,1]}$ for narrow/single composition cases. Similarly, the residual is high is the true input is a broad growth factor frequency distribution that is attempted to be fitted using $L S Q_{1}$. For example, the red spectrum in the left graph of Fig. 4 shows poor agreement with the input and results in a much larger residual than $L S Q_{1}$ (values not shown). Therefore, a proposed unsupervised inversion scheme is to compute the solution of $L S Q_{1}, L S Q_{2}$ and $L_{0} D_{1 e-3} B_{[0,1]}$ and then select the solution with the lowest residual relative to the apparent growth factor distribution.

A limitation of the above approach is that the forward model (and thus matrix $\mathbf{A}_{2}$ ) assumes that the larger multiply charged particles have the same growth factor frequency distribution as the smaller singly charged particles. This limitation can in principle be eliminated by specifying a 2D probability frequency distribution that also depends on dry diameter. Constructing an appropriate forward model that adds another integration dimension to Eq. (14) is straightforward. An inversion that solves for the 2D frequency distribution, similar to those performed elsewhere (Rawat et al., 2016; Sipkens et al., 2020), is feasible using the algorithms in RegularizationTools.jl and has been attempted by the author. In practice, however, this approach proved impractical. For example, using 10 dry diameters and a 30-bin size resolution results in a large inversion matrix. Adding an integration dimension to the forward model and recomputing this matrix for each scan significantly slows the inversion. Furthermore, interpolation is needed to estimate the growth factor frequency distribution for the multiply charged particles. The physical size of the multiply charged particles depends on their charge. For example, +2 charged particles are approximately 1.5 times larger than +1 charged particles. The diameter of the multiply charged particles will therefore not necessarily coincide with any of the 10 dry diameters selected for direct measurement. This introduces additional uncertainty due to assumptions that need to be made in the interpolation scheme. Errors from scans with low concentration at the edge of the size distribution propagate back into the inversion at other dry sizes. Finally, only a single size distribution can be used to compute the matrix $\mathbf{A}_{2}$. Collecting data for 10 dry sizes can take 20 min or longer, during which the aerosol size distribution may change, thus 
invalidating the use of a single inversion matrix. Although no exhaustive analysis was performed, the compounding errors during a $2 \mathrm{D}$ inversion seem to outweigh the benefits of relaxing the assumption that +2 and +3 charged particles have the same growth factor frequency distribution as the +1 charged particles.

\section{Inversion of Real-World Data}

\subsection{Data Sources}

\subsubsection{Bodega Bay Marine Laboratory}

Aerosol size distribution data to contrast inversion schemes were obtained from measurements taken at Bodega Bay Marine Laboratory $\left(39^{\circ} 18^{\prime} 25^{\prime \prime} \mathrm{N} 123^{\circ} 3^{\prime} 58^{\prime \prime} \mathrm{W}\right)$ between 16 January 2015 and 8 March 2015 as part of the Calwater 2/ACAPEX campaign. A subset of the data have been published by Atwood et al. (2019). Sample flow was brought into a mobile laboratory using an inlet, dried to $10 \pm 2 \%$ relative humidity using a Nafion membrane drier, and brought to charge equilibrium using an X-ray source (TSI 3088, TSI Inc., Shoreview, MN, U.S.A.) prior to entering a cylindrical DMA column (TSI 3080). The DMA was configured to measure the size distribution in scanning mobility particle sizer mode. Voltage was scanned exponentially from $10 \mathrm{kV}$ to $10 \mathrm{~V}$ over $300 \mathrm{~s}$. A condensation particle counter (TSI 3771, flow rate $=1 \mathrm{~L} \mathrm{~min}^{-1}$ ) and a cloud condensation nuclei counter (DMT Model 100, Droplet Measurement Technologies, Boulder, CO, U.S.A., flow rate $=0.3 \mathrm{~L} \mathrm{~min}^{-1}$ ) were used to measure particle concentration downstream of the DMA. The sheath-to-sample flow rate in the DMA was 5:1.3 $\mathrm{L} \mathrm{min}^{-1}$. Raw DMA response distributions comprising CPC concentration vs. apparent +1 mobility diameter were constructed along a 120-bin, geometrically-stepped mobility grid. Response distributions are denoted as $\mathbb{r}$. The apparent +1 mobility diameter is computed from the centroid mobility selected by the DMA assuming that all particles are singly charged. The dynamic diameter range for this setup is from 12 to $550 \mathrm{~nm}$. The inversion matrix $\mathbf{A}$ is computed using Eq. (10) for the diffusionallybroadened transfer function (Stolzenburg and McMurry, 2008) and transmission loss correction through the DMA (Reineking and Porstendörfer, 1986). Inclusion of these terms results in a more ill-posed inverse problem due to the addition of small terms in the matrix A. The DMA response functions were inverted using the $L_{0} x_{0} B_{[0, \infty]}$ and $L_{2} B_{[0, \infty]}$ methods. The $a$ priori estimate for $L_{0} x_{0} B_{[0, \infty]}$ was taken to be $\mathrm{x}_{0}=\mathbf{S}^{-1} \mathbb{r}$, where $\mathbf{S}$ is obtained by summing the rows of $\mathbf{A}$ and placing the results on the diagonal of $\mathbf{S}$ (Talukdar and Swihart, 2003). The method $L_{0} x_{0} B_{[0, \infty]}$ with $\mathrm{x}_{0}=\mathbf{S}^{-1} \mathrm{r}$ is essentially equivalent to the method used by Petters (2018), where it was shown that the thus-inverted spectra are similar to those output by the inversion algorithm employed by the commercial TSI Aerosol Instrument Manager software suite. Small differences between $L_{0} x_{0} B_{[0, \infty]}$ employed here and the approach of Petters (2018) include the use of generalized cross-validation instead of the L-curve method to search for the optimal regularization parameter and the method to eliminate negative values after inversion. Petters (2018) truncated negative values instead of using a least squares numerical solver as described in section 2.1.3. 


\subsubsection{Southern Great Plains Site}

Aerosol size distribution and humidified tandem DMA data to illustrate the tandem DMA inversion schemes were taken from measurements made by the U.S. Department of Energy (DOE) Atmospheric Radiation Measurement (ARM) program. The Southern Great Plains (SGP) site is located in Lamont, OK, U.S.A. (Lat: 36.604937, Long:-97.485561) and placed in a rural continental setting that is surrounded by agricultural activity as well as oil and gas production. The aerosol evolution at the site is influenced by frequent new particle formation events (Hodshire et al., 2016; Chen et al., 2018; Marinescu et al., 2019). Number concentrations fluctuate in response to the nitrate and organic aerosol cycle on short time scales and synoptic weather variability on longer time scales. During winter months, the inorganic aerosol composition at the site is dominated by nitrate aerosols (Jefferson et al., 2017; Mahish et al., 2018) and hygroscopicity derived from scattering measurements is largest during those months (Jefferson et al., 2017).

The instruments/measurements are part of the Aerosol Observing System (AOS, Uin and Smith, 2020). The instruments are operated by DOE personnel and data are distributed through a publicly accessible archive. Size distributions were measured with a scanning mobility particle sizer (TSI Model 3936, Kuang, 2016). Data in the archive are already inverted and reported at 5-min intervals. Humidified DMA response functions were measured using a humidified tandem DMA (Model 3100, Brechtel

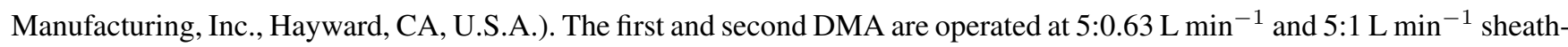
to-sample flow ratio, respectively (Janek Uin, personal communication). The instrument measures the humidified mobility distribution function at $85 \%$ relative humidity for 50,100, 150, 200, and $250 \mathrm{~nm}$ dry diameter particles. Typical data density results in 228 scans per day, with equal coverage for the five dry sizes. Pre-processing that is already applied to the archived data accounts for conversion between mobility and apparent mobility diameter, the size-dependent detector counting efficiency, and number count smearing during the scan resulting from insufficient particle counter response time. When divided by the dry diameter, the archived data correspond to the apparent growth factor distribution evaluated by the forward model in Eq. (15).

The matrix $\mathbf{A}_{2}$ was evaluated for each scan using the flow rates given above, the dimensions of the DMAs given in LopezYglesias et al. (2014), and the aerosol size distribution measured by the co-located SMPS with the timestamp closest to the a scan of the humidified tandem DMA. Typical time differences between the two instruments' scan times are between 1 and 3 min. The humidified size distribution was interpolated onto a discrete growth factor grid with 30 bins to match the matrix $\mathbf{A}_{2}$. The data were then inverted using the $L_{0} D_{1 e-3} B_{[0,1]}$ method. The method $L_{0} D_{1 e-3} B_{[0,1]}$ was further constrained such that growth factors $<1$ are disallowed. This is achieved by setting the upper bound to zero for bins with $g f<1$. Growth factors less than unity can occur due to particle restructuring upon humidification (Mikhailov et al., 2004; Shingler et al., 2016) or evaporation during transit through the humidifier and second DMA. Both effects are assumed to be less important for ambient aerosol compared to the desire to constrain the inversion. In addition, the efficacy of the $L S Q_{1}$ and $L S Q_{2}$ methods for inverting the data was tested. For each scan, the root mean square error between the measured apparent growth factor distribution and the predicted growth factor distribution was evaluated for all three inversion approaches $\left(L_{0} D_{1 e-3} B_{[0,1]}, L S Q_{1}\right.$, and $\left.L S Q_{2}\right)$. The method that resulted in the smallest residual was taken to be the inverted growth factor frequency distribution. 

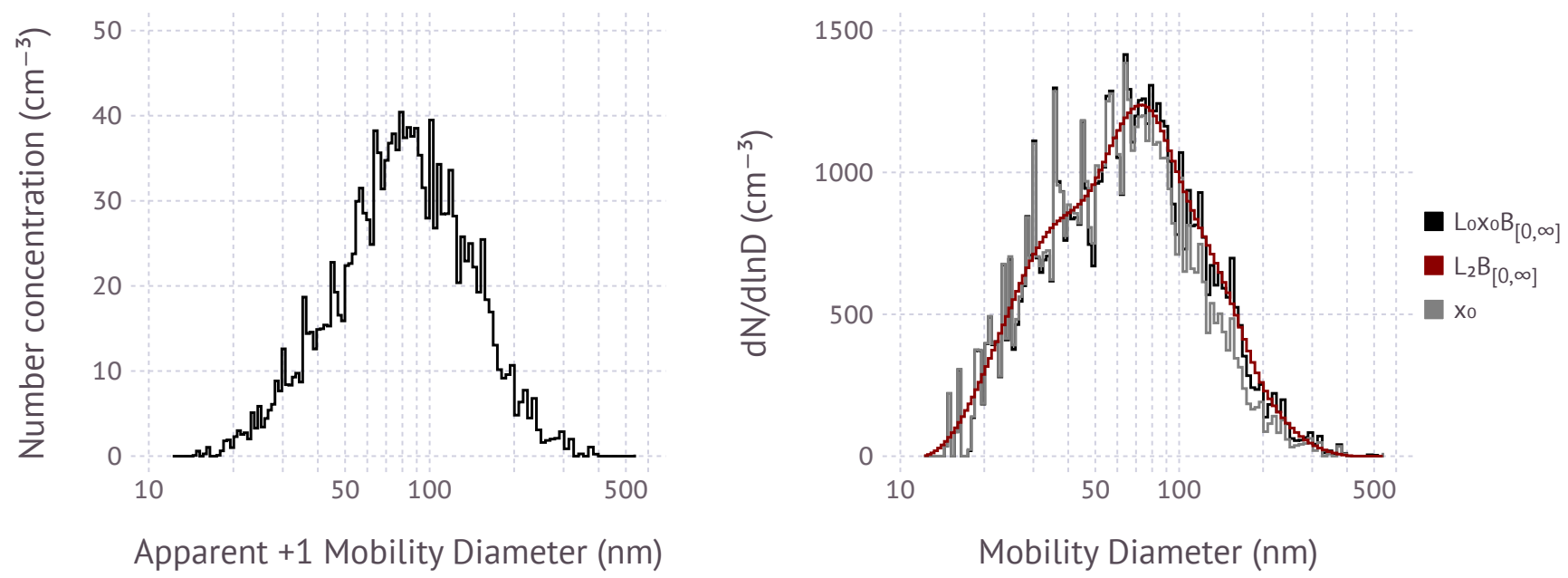

Figure 5. Left: Raw DMA response function for a single scan on 5 March 2015 at 10:40 UTC at Bodega Bay Marine Laboratory. Right: Inverted size distribution using the $L_{0} x_{0} B_{[0, \infty]}$ and $L_{2} B_{[0, \infty]}$ method and the a-priori estimate of the solution $x_{0}=\mathbf{S}^{-1} \mathrm{r}$.

\subsection{Results}

\subsubsection{Inversion of Size Distribution Data (Bodega Bay Marine Laboratory Site)}

Figure 5 shows a real-world example size distribution response function gridded into 120 size bins. The total particle concentration is $\approx 2000 \mathrm{~cm}^{-3}$. The ragged structure is typically explained by random noise due to Poisson counting statistics. At this diameter resolution, and with inclusion of the diffusion and loss terms in the forward model, the unregularized matrix inverse is entirely dominated by amplified random noise and is useless. The $L_{0} x_{0} B_{[0, \infty]}$ method converges to the solution with slight amplification of the random noise presented in the raw response function. The random noise is carried over into the a-priori estimate $\mathrm{x}_{0}=\mathbf{S}^{-1} \mathbb{r}$, which roughly represents the noise visible in the reconstructed solution. Nevertheless, $L_{0} x_{0} B_{[0, \infty]}$ is highly robust and unlikely to go astray, because $\mathrm{x}_{0}$ is an excellent approximation of the solution at diameters less than 100 $\mathrm{nm}$ where singly charged particles dominate and is a good initial estimate for larger particles. Second order inversion using $L_{2} B_{[0, \infty]}$ produces a smooth, denoised solution due to application of the derivative operator in the regularization filter matrix. The solution converges even though no a-priori estimate is used, i.e., $\mathrm{x}_{0}=0$. The algorithms specified in Section 2.1.2 significantly speed up the inversion relative to previous versions of the software (Petters, 2018). Wall-clock times on an i7-8559U CPU for the inversion of a single spectrum are 5 and $2 \mathrm{~ms}$ for $L_{0} x_{0} B_{[0, \infty]}$ and $L_{2} B_{[0, \infty]}$, respectively. This contrasts to 500 to $1000 \mathrm{~ms}$ required by the brute force algorithm - approximately equivalent to $L_{0} x_{0} B_{[0, \infty]}$ - used previously. Finding the global minimum of $V(\lambda)$ to identify the optimal regularization parameter also eliminates the occasional failure to converge when the L-curve algorithm is used (Petters, 2018). Either $L_{0} x_{0} B_{[0, \infty]}$ or $L_{2} B_{[0, \infty]}$, combined with generalized cross-validation, are suitable to be used in routine unsupervised inversion of size distribution data. 


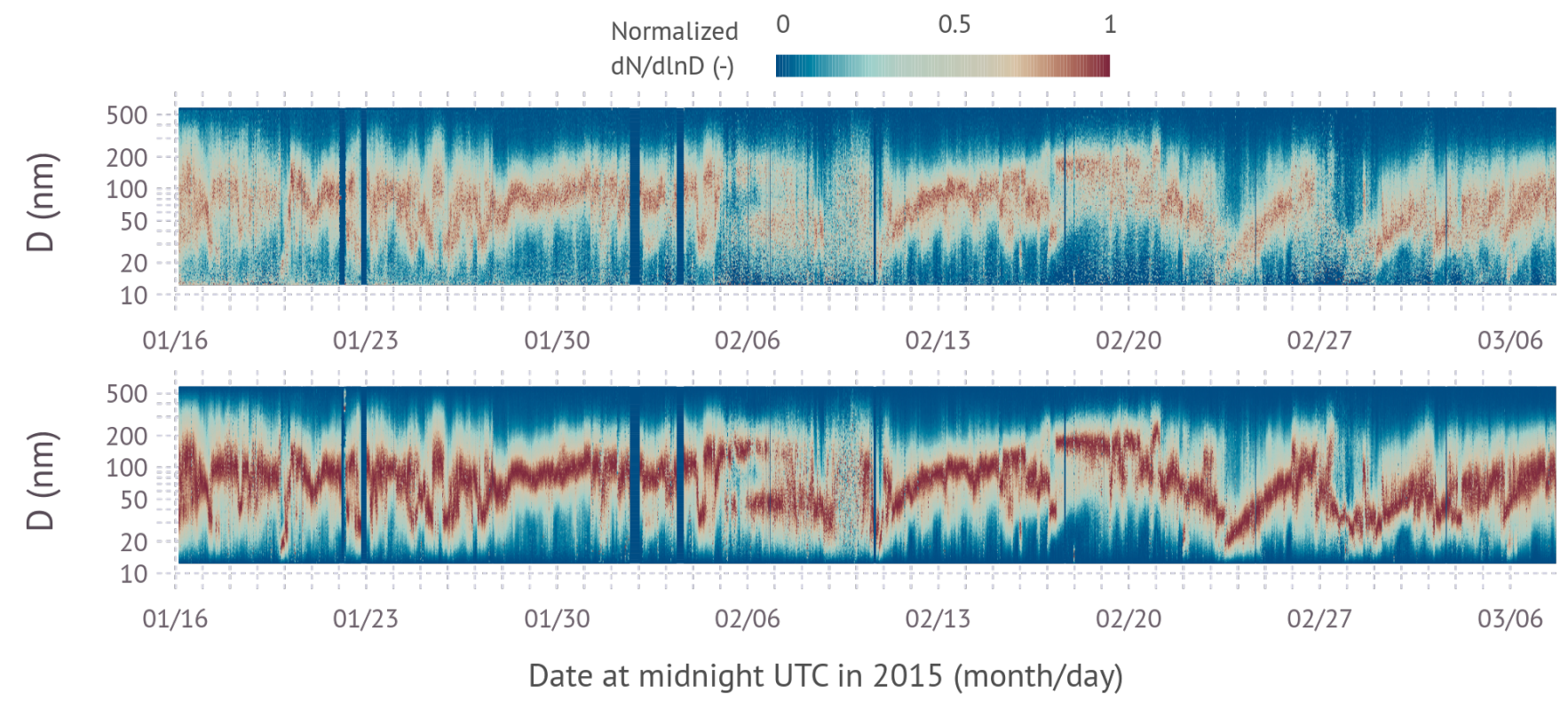

Figure 6. Time evolution of the normalized particle size distributions collected between 16 January and 7 March at Bodega Bay Marine Laboratry. The normalization is for each size distribution such that the maximum of the spectral density equals to unity. The red color visualizes the time evolution of the mode diameter of the dominant mode. Top panel: inverted using $L_{0} x_{0} B_{[0, \infty]}$; bottom panel: inverted using $L_{2} B_{[0, \infty]}$.

Figure 6 shows the time evolution of the normalized particle size distributions over a seven week period. The normalization is to highlight changes in the mode diameter(s). In general, the aerosol at the site is dominated by continental rural background conditions and the land-sea breeze circulation (Atwood et al., 2019). The timeseries is punctuated by aerosol transported from the California Central Valley to the site through the Petaluma Gap (Martin et al., 2017). Periods of low particle concentration occurred during the passage of an atmospheric river on 7-9 February 2015 and a marine inflow event on March $27-282015$.

The atmospheric river brought heavy precipitation and marine airmasses from the southwest direction, while the marine inflow event brought strong winds and precipitation free maritime air from the northwest direction. Several periods of prolonged modal growth were observed starting, e.g., 9 February 2015, 24 February 2015, and 29 February 2015. Figure 6 demonstrates the influence of inversion noise on visualizing the dynamic evolution of the size distribution. The denoised $L_{2} B_{[0, \infty]}$ solution significantly improves visualization of modes without the need to reduce the size resolution in the inversion. The signal is especially improved during low concentration periods during the atmospheric river passage and marine inflow event.

\subsubsection{Inversion Humidified Tandem DMA Data (DOE ARM SGP Site)}

Figure 7 shows real-world examples of growth factor frequency distributions for five dry sizes. Also shown for context is the evolution of the normalized aerosol number size distribution. Figure 7 shows dynamic evolution of the size distribution 
https://doi.org/10.5194/amt-2021-51

Preprint. Discussion started: 17 March 2021

(C) Author(s) 2021. CC BY 4.0 License.
Atmospheric Measurement Techniques

Discussions

Colorscale $\begin{array}{lll}0 & 0.5 & 1\end{array}$

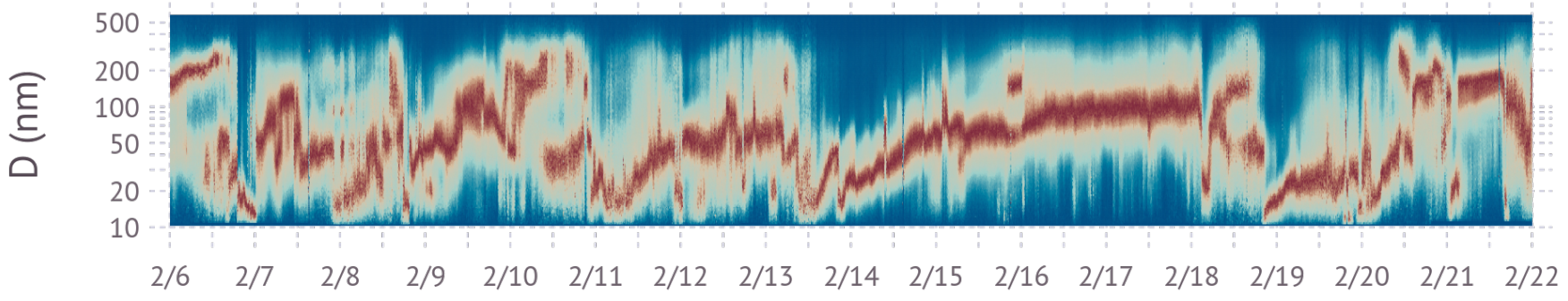

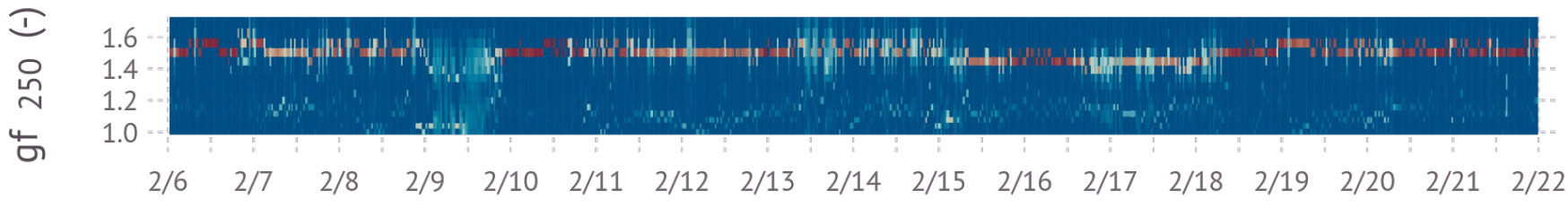
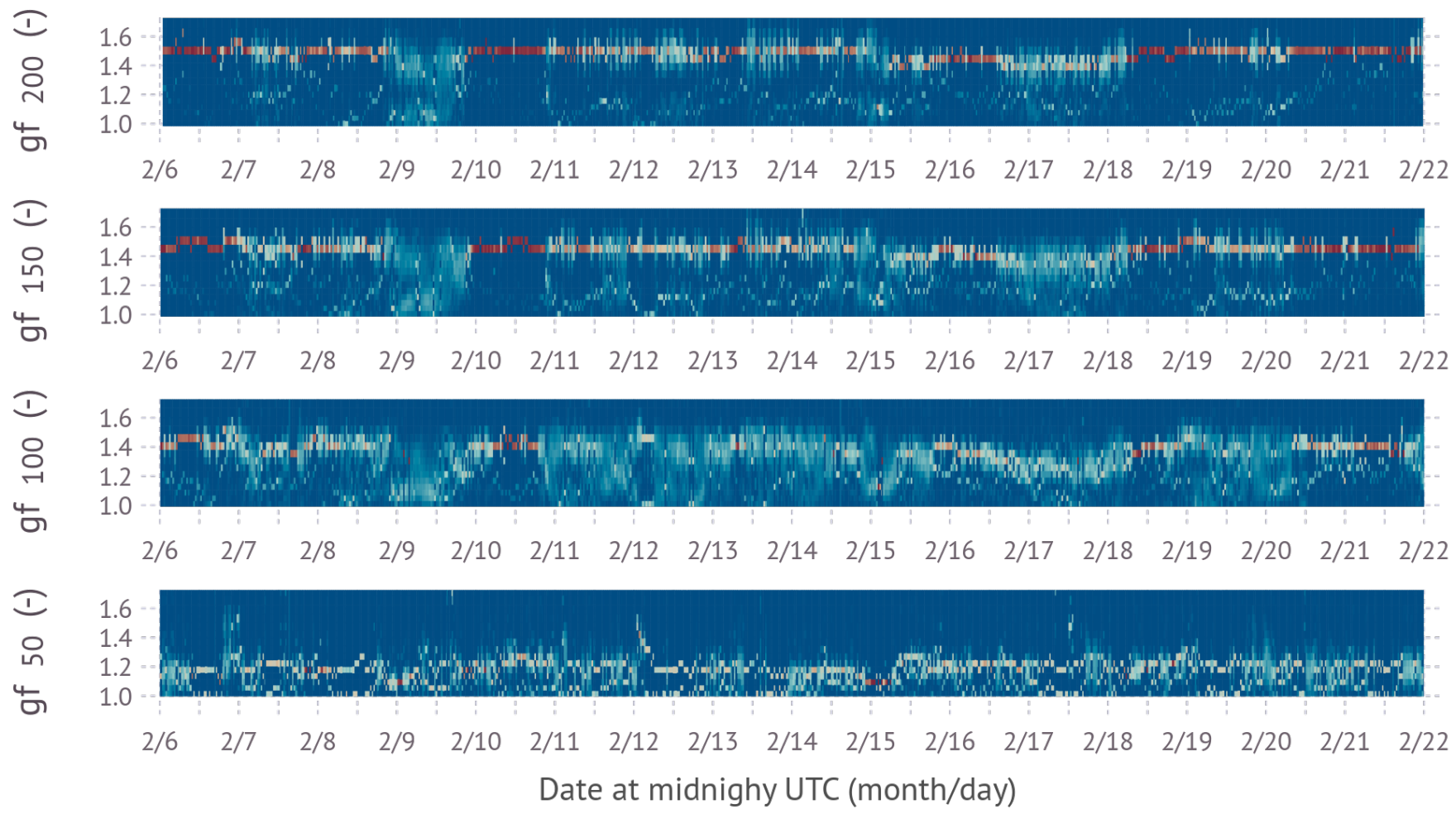

Figure 7. Top: Time evolution of the normalized particle size distributions collected between 6 February and 22 February at the Southern Great Plains research site. The normalization is for each size distribution such that the maximum of the spectral density equals to unity. Second-Top to Bottom: Inverted growth factor frequency distributions at $85 \%$ relative humidity for 250, 200, 150, 100, and 50 nm particles, respectively. 
with sudden changes in mode diameter, several apparent new particle formation events, and several prolonged modal growth events. The distribution of the methods selected for best inversion was $L S Q_{1}$ ( $\sim 5 \%$ of spectra), $L S Q_{2}$ ( $\sim 50 \%$ of spectra), and $L_{0} D_{1 e-3} B_{[0,1]}\left(\sim 45 \%\right.$ of spectra). In Fig. 7, the $L S Q_{2}$ inverted frequency distributions show a clean bimodal structure ( 2 colors per scan), while the $L_{0} D_{1 e-3} B_{[0,1]}$ spectra appear more smeared. The $250 \mathrm{~nm}$ dry diameter data show a dominant contribution of more hygroscopic particles with $g f \sim 1.5-1.6$ and a small contribution of less hygroscopic particles with $g f$ $\sim 1.05-1.2$. Similar trends are observed for 200,150 , and $100 \mathrm{~nm}$ particles. However, the hygroscopicity of the dominant mode decreases with decreasing diameter. The fraction of cases where a broad hygroscopicity frequency distribution is observed is larger than for the $250 \mathrm{~nm}$ particles. Notably, time periods with broad growth factor frequency distributions are observed at multiple sizes. For example, the period of $9-11$ February 2020 shows a broad frequency distribution at 100, 150, 200, and $250 \mathrm{~nm}$ dry diameter. Occasionally temporal trends in the hygroscopicity of the less hygroscopic mode are observed. For example, the growth factor of the less hygroscopic mode systematically increases on 20 February 2020 for 150, 200, and 250 $\mathrm{nm}$ particles, indicative of a chemical transformation of some, but not all, of the particles. The $50 \mathrm{~nm}$ dry particle hygroscopicity frequency distributions are also predominantly bimodal. However, the overall growth factor is significantly smaller, with most $g f<1.2$.

\section{Discussion, Summary, and Conclusions}

RegularizationTools.jl is a general purpose software package to invert data using $L_{2}$-regularization. It is included as a supplement to this work and published as free software through the GNU General Public License. The package implements well-established numerical algorithms (Golub et al., 1979; Eldén, 1982; Bates et al., 1986; Hansen, 1998, 2000; Mogensen and Riseth, 2018) and filter matrices (Huckle and Sedlacek, 2012). Systems with up to $~ 1000$ equations can be inverted. The upper limit is determined by the need to compute the generalized singular value decomposition of the design matrix and filter matrix, which has at minimum $O\left(n^{2}\right)$ time complexity. The time to compute the generalized singular value decomposition exceeds several 10s of seconds for systems exceeding 1000 equations. Iterative methods to support inversion of large-scale systems have been formulated (e.g. Lampe et al., 2012), but these are currently not implemented.

The software package can be used to simplify the prototyping of a wide variety of inverse problems that arise in science and engineering applications. Although the package does not add any novel regularization methods, it provides a systematic method to categorize inversion methods via the expression in Eq. (5). A total of 24 basic permutations can be combined with a set of hyperparameters to attempt the inversion of ill-posed problems. Hyperparameters include boundary constraints, values for a-priori estimates, and the lower bound $\epsilon$ for the Huckle and Sedlacek (2012) two-pass inversion approach. Users can define custom filter matrices and thus are able to further extend the number of methods. Equation (7) provides an example of a simplified interface that allows testing of different permutations with a simple function call. Furthermore, a generic interface is provided to translate arbitrary linear forward models defined by a computer function into the corresponding matrix of linear transformation. This obviates the need to explicitly write out the Fredholm integral equation and discretize it using the quadrature or the Galerkin method. For example, the forward model for transfer of a growth factor frequency distribution 
through the tandem DMA in Eq. (14) represents a triple integral and also contains a sum term for the multiple charges. Explicit discretization of this model would be tedious compared to the method employed here. As demonstrated in the documentation of the package, the generic interface can readily be used to solve other common inversion problems. Only a few lines of new code are needed to reproduce the essential core of the algorithm used in the unsupervised inversion of Lidar data (Müller et al., 2019), which involves the retrieval of a size distribution from multi-wavelength scattering and absorption data (see package documentation for code).

$L_{2}$-regularization has been used previously for size-distribution inversion (e.g. Wolfenbarger and Seinfeld, 1990; Talukdar and Swihart, 2003; Petters, 2018). However, to the author's knowledge the package DifferentialMobilityAnalyzers.jl is the only publicly available free-software for size distribution inversion from DMA data. This work extends the capabilities of that package. The $L_{0} x_{0} B_{[0, \infty]}$ and $L_{2} B_{[0, \infty]}$ methods can be used with generalized cross-validation to perform fast unsupervised inversion of size distribution data. Convergence issues resulting from the use of the L-curve method used previously (Petters, 2018) are resolved by switching to the generalized cross-validation approach to find the optimal regularization parameter. Higher-order inversions resulting in smooth, denoised solutions are now available. It is expected that such denoised spectra will benefit unsupervised machine-learning approaches that seek to extract features from such datasets (e.g. Joutsensaari et al., 2018; Atwood et al., 2019), although this hypothesis has not been tested by the author. Revision of the numerical algorithms improves the speed of inversion by a factor $\sim 200$. The millisecond inversion speed for a single scan permits rapid inversion of large datasets and facilitates inversion in real-time during data acquisition on low-cost and low computational power hardware platforms. For example, the inversion has been tested on ARM Cortex A72/A53 64 bit reduced-instruction-set architecture used by the ROCKPro64 single board computer. The Julia language provides tier 1 support for this architecture. Julia binaries are available; DifferentialMobilitityAnalyzers.jl and RegularizationTools.jl compile and run without any modification. Inversion speeds on the order of several 10s of milliseconds are fast enough on this inexpensive but relatively low-powered platform to permit embedding the inversion into the data acquisition and display software and running the inversion before each display update.

To the author's knowledge this is the first time $L_{2}$-regularization has been applied to the inversion of tandem DMA data. Inversion of simulated data shows that the an SMPS-style matrix-based inversion is possible, while also accounting for multiply charged particles. Application of solution constraints fixes the issue of oscillatory and negative solutions that were encountered with the matrix-based optimal estimation method used by Cubison et al. (2005). The 12 methods that include boundary constraints were systematically tested against five test cases. All of the methods performed similarly well when inverting frequency distributions. However, poor results were obtained when inverting narrow distributions or data produced by single compositions. The method $L_{0} D_{1 e-3} B_{[0,1]}$ is often, but not always, able to invert these data. For narrow distributions a nonlinear least squares fit with either one or two growth factors, termed $L S Q_{1}$ and $L S Q_{2}$, can fill this gap. Ambient data can be inverted by applying all three methods and then selecting the inversion with the smallest root mean square error between the data and the prediction. In contrast to previous inversion routines (Stolzenburg and McMurry, 1988; Cubison et al., 2005; Gysel et al., 2009), explicit knowledge of the aerosol size distribution is needed. These data can either be obtained using a co-located scanning mobility particle sizer, or by configuring the tandem DMA to also measure the size distribution every few scans. 
The resulting algorithm is unsupervised and nonparametric, i.e., it can be fully automated and does not require any a-priori assumption about the shape of the growth factor frequency distribution. The speed of the inversion algorithm is much slower than for size distribution inversion for several reasons. For each scan, the matrix $\mathbf{A}_{2}$ must be recomputed to account for changes in the size distribution. This requires recomputing the generalized singular value decomposition for $\mathbf{A}_{2}$ and $\mathbf{L}$, which is slow. Furthermore, three inversions are computed for each scan. The $L S Q_{1}$ and $L S Q_{2}$ methods use a gradient descent algorithm together with the forward model, which is slower than the matrix inverse. Nevertheless, a single day's worth of data can be inverted on a regular personal computer within a few minutes.

Application of the inversion to a 16-day dataset demonstrates that the thus-obtained growth factor frequency distribution data can reveal significant details about the mixing state of the aerosol. The inverted dataset is suitable as input to carry out common analyses made with growth factor frequency distributions, including characterization of the evolution of aerosol mixing state as a function of time, characterization of changes in growth factor with dry diameter and its relationship to chemical composition, characterization of the growth factor at the mode diameter of particles during modal growth events (Park et al., 2008; Wu et al., 2013; Jung and Kawamura, 2014), decomposition of the hygroscopicity frequency distributions into distinct growth factor classes (Swietlicki et al., 2008), evaluation of the temporal trends of spectral concentration for hygroscopicity-resolved data (Royalty et al., 2017), evaluation of the accuracy of aerosol mass spectrometer measured (organic) mass concentration through hygroscopicity constraints (Jimenez et al., 2016), and inclusion of growth factor frequency distributions to account for mixing state in aerosol hygroscopicity to cloud condensation nuclei closure (Mahish et al., 2018).

\section{Code and data availability.}

1. SGP SMPS Data: Atmospheric Radiation Measurement (ARM) user facility. 2016, updated hourly. Scanning mobility particle sizer (AOSSMPS). 2020-01-01 to 2020-09-27, Southern Great Plains (SGP) Lamont, OK (Extended and Co-located with C1) (E13). Compiled by C. Kuang, C. Salwen, M. Boyer and A. Singh. ARM Data Center. Data set accessed 2020-09-29 at http://dx.doi.org/10.5439/ 1095583.

2. SGP HTDMA Data: Atmospheric Radiation Measurement (ARM) user facility. 2017, updated hourly. Humidified Tandem Differential Mobility Analyzer (AOSHTDMA). 2020-01-01 to 2020-02-22, Southern Great Plains (SGP) Lamont, OK (Extended and Co-located with C1) (E13). Compiled by J. Uin, C. Salwen and G. Senum. ARM Data Center. Data set accessed 2020-09-25 at http://dx.doi.org/ $10.5439 / 1095581$.

3. Bodega Bay Preprocessed Data: Petters, Markus D., Rothfuss, Nicholas E., Taylor, Hans, Kreidenweis, Sonia M., DeMott, Paul J., and Atwood, Samuel A. (2019). Size-resolved cloud condensation nuclei data collected during the CalWater 2015 field campaign (Version v1.0) [Data set]. Zenodo. http://doi.org/10.5281/zenodo.2605668.

4. DifferentialMobilityAnalyzers.jl: A general purpose software package implementing the "Language to Simplify Computation of Differential Mobility Analyzer Response Functions" is available using the GPL-v3 license and is hosted is on GitHub. Version 2.5 was used in this work. The version of the software will be permanently archived with a doi upon acceptance of the manuscript for publication. 
https://doi.org/10.5194/amt-2021-51

Preprint. Discussion started: 17 March 2021

(c) Author(s) 2021. CC BY 4.0 License.

(c) (i)
Atmospheric

Measurement

Techniques

Discussions

Documentation: https://mdpetters.github.io/DifferentialMobilityAnalyzers.j1/stable/

Source Code: https://github.com/mdpetters/DifferentialMobilityAnalyzers.j1

5. RegularizationTools.jl: A general purpose software package implementing Phillips-Twomey-Tikhonov Regularization. The package is available using the GPL-v3 license and source code and documentationa are hosted on GitHub. Version 0.4.0 was used in this work.

The version of the software will be permanently archived with a doi upon acceptance of the manuscript for publication.

Documentation: https://mdpetters.github.io/RegularizationTools.j1/stable/

Source Code: https://github.com/mdpetters/RegularizationTools.jl

6. Source Code to reproduce the figures and derived datasets, and copies of the derived dartasets are temporarily hosted on GitHub and will be permanently archived on Zenodo with a doi upon final acceptance of the manuscript for publication. Source Code: https://github.com/mdpetters/softwarePackageSimplify2021

Competing interests. The author declares no competing interests.

Acknowledgements. This work was supported by the United States Department of Energy, Office of Science, Biological and Environment Research, grant number DE-SC 0021074 and NASA grant number 80NSSC19K0124. Data from the SGP site were obtained from the Atmospheric Radiation Measurement (ARM) Program sponsored by the U.S. Department of Energy, Office of Science, Office of Biological and Environmental Research, Climate and Environmental Sciences Division. I thank Janek Uin for providing additional information about the data. Size distribution data at Bodega Bay Marine Laboratory were collected with support from the National Science Foundation grant AGS-1450690. I thank Nicholas Rothfuss, Sam Atwood, and Hans Taylor for help operating the SMPS at Bodega Bay Marine Laboratory. I thank Kimberly Prather, Sonia Kreidenweis, and Paul DeMott for logistical support during the field campaign. I thank Sarah Petters for helpful discussions. 
Agarwal, S., Mierle, K., and Others: Ceres Solver, http://ceres-solver.org.

Atwood, S. A., Kreidenweis, S. M., DeMott, P. J., Petters, M. D., Cornwell, G. C., Martin, A. C., and Moore, K. A.: Classification of Aerosol Population Type and Cloud Condensation Nuclei Properties in a Coastal California Littoral Environment Using an Unsupervised Cluster Model, Atmos. Chem. Phys., 19, 6931-6947, https://doi.org/10.5194/acp-19-6931-2019, 2019.

Baart, M. L.: The Use of Auto-Correlation for Pseudo-Rank Determination in Noisy III-Conditioned Linear Least-Squares Problems, IMA Journal of Numerical Analysis, 2, 241-247, https://doi.org/10.1093/imanum/2.2.241, 1982.

Bates, D. M., Lindstrom, M. J., Wahba, G., and Yandell, B. G.: GCVPACK - Routines for Generalized Cross Validation, Tech. Rep. Technical Report No. 775, University of Wisconsin, Department of Statistics, 1986.

Bezanson, J., Edelman, A., Karpinski, S., and Shah, V. B.: Julia: A Fresh Approach to Numerical Computing, SIAM Review, 59, 65-98, https://doi.org/10.1137/141000671, 2017.

Borsdorff, T., Hasekamp, O. P., Wassmann, A., and Landgraf, J.: Insights into Tikhonov Regularization: Application to Trace Gas Column Retrieval and the Efficient Calculation of Total Column Averaging Kernels, Atmospheric Measurement Techniques, 7, 523-535, https://doi.org/10.5194/amt-7-523-2014, 2014.

Chen, H., Hodshire, A. L., Ortega, J., Greenberg, J., McMurry, P. H., Carlton, A. G., Pierce, J. R., Hanson, D. R., and Smith, J. N.: Vertically Resolved Concentration and Liquid Water Content of Atmospheric Nanoparticles at the US DOE Southern Great Plains Site, Atmospheric Chemistry and Physics, 18, 311-326, https://doi.org/10.5194/acp-18-311-2018, 2018.

Cubison, M., Coe, H., and Gysel, M.: A Modified Hygroscopic Tandem DMA and a Data Retrieval Method Based on Optimal Estimation, Journal of Aerosol Science, 36, 846-865, https://doi.org/10.1016/j.jaerosci.2004.11.009, 2005.

Dawson, K. W., Petters, M. D., Meskhidze, N., Petters, S. S., and Kreidenweis, S. M.: Hygroscopic Growth and Cloud Droplet Activation of Xanthan Gum as a Proxy for Marine Hydrogels, Journal of Geophysical Research: Atmospheres, 121, 11,803-11,818, https://doi.org/10.1002/2016JD025143, 2016.

Dubovik, O. and King, M. D.: A Flexible Inversion Algorithm for Retrieval of Aerosol Optical Properties from Sun and Sky Radiance Measurements, Journal of Geophysical Research: Atmospheres, 105, 20 673-20 696, https://doi.org/10.1029/2000JD900282, 2000.

Eldén, L.: A Weighted Pseudoinverse, Generalized Singular Values, and Constrained Least Squares Problems, BIT Numerical Mathematics, 22, 487-502, https://doi.org/10.1007/BF01934412, 1982.

Farmer, D. K., Cappa, C. D., and Kreidenweis, S. M.: Atmospheric Processes and Their Controlling Influence on Cloud Condensation Nuclei Activity, Chemical Reviews, 115, 4199-4217, https://doi.org/10.1021/cr5006292, 2015.

Golub, G. H., Heath, M., and Wahba, G.: Generalized Cross-Validation as a Method for Choosing a Good Ridge Parameter, Technometrics, 21, 215-223, https://doi.org/10.2307/1268518, 1979.

Gysel, M., McFiggans, G., and Coe, H.: Inversion of Tandem Differential Mobility Analyser (TDMA) Measurements, Journal of Aerosol Science, 40, 134-151, https://doi.org/10.1016/j.jaerosci.2008.07.013, 2009.

Hansen, P. C.: 2. Decompositions and Other Tools, in: Rank-Deficient and Discrete Ill-Posed Problems, Mathematical Modeling and Computation, pp. 19-44, Society for Industrial and Applied Mathematics, https://doi.org/10.1137/1.9780898719697.ch2, 1998.

Hansen, P. C.: The L-Curve and Its Use in the Numerical Treatment of Inverse Problems., in: Advances in Computational Bioengineering, edited by Johnston, P., pp. 119-142., WIT Press., 2000. 
https://doi.org/10.5194/amt-2021-51

Preprint. Discussion started: 17 March 2021

(c) Author(s) 2021. CC BY 4.0 License.

(c) (i)

Atmospheric

Measurement

Techniques

Discussions

Hansen, P. C.: Regularization Tools Version 4.0 for Matlab 7.3, Numerical Algorithms, 46, 189-194, https://doi.org/10.1007/s11075-0079136-9, 2007.

Hodshire, A. L., Lawler, M. J., Zhao, J., Ortega, J., Jen, C., Yli-Juuti, T., Brewer, J. F., Kodros, J. K., Barsanti, K. C., Hanson, D. R., McMurry, P. H., Smith, J. N., and Pierce, J. R.: Multiple New-Particle Growth Pathways Observed at the US DOE Southern Great Plains Field Site, Atmospheric Chemistry and Physics, 16, 9321-9348, https://doi.org/10.5194/acp-16-9321-2016, 2016.

Huckle, T. and Sedlacek, M.: Data Based Regularization Matrices for the Tikhonov-Phillips Regularization, PAMM, 12, 643-644, https://doi.org/10.1002/pamm.201210310, 2012.

Jefferson, A., Hageman, D., Morrow, H., Mei, F., and Watson, T.: Seven Years of Aerosol Scattering Hygroscopic Growth Measurements from SGP: Factors Influencing Water Uptake, Journal of Geophysical Research: Atmospheres, 122, 9451-9466, https://doi.org/10.1002/2017JD026804, 2017.

Jiang, J., Kim, C., Wang, X., Stolzenburg, M. R., Kaufman, S. L., Qi, C., Sem, G. J., Sakurai, H., Hama, N., and McMurry, P. H.: Aerosol Charge Fractions Downstream of Six Bipolar Chargers: Effects of Ion Source, Source Activity, and Flowrate, Aerosol Science and Technology, 48, 1207-1216, https://doi.org/10.1080/02786826.2014.976333, 2014.

Jimenez, J. L., Canagaratna, M. R., Drewnick, F., Allan, J. D., Alfarra, M. R., Middlebrook, A. M., Slowik, J. G., Zhang, Q., Coe, H., Jayne, J. T., and Worsnop, D. R.: Comment on "The Effects of Molecular Weight and Thermal Decomposition on the Sensitivity of a Thermal Desorption Aerosol Mass Spectrometer”, Aerosol Science and Technology, 50, i-xv, https://doi.org/10.1080/02786826.2016.1205728, 2016.

Joutsensaari, J., Ozon, M., Nieminen, T., Mikkonen, S., Lähivaara, T., Decesari, S., Facchini, M. C., Laaksonen, A., and Lehtinen, K. E. J.: Identification of New Particle Formation Events with Deep Learning, Atmospheric Chemistry and Physics, 18, 9597-9615, https://doi.org/10.5194/acp-18-9597-2018, 2018.

Jung, J. and Kawamura, K.: Hygroscopic Properties of Newly Formed Ultrafine Particles at an Urban Site Surrounded by Deciduous Forest (Sapporo, Northern Japan) during the Summer of 2011, Atmos. Chem. Phys., 14, 7519-7531, https://doi.org/10.5194/acp-14-7519-2014, 2014.

Kandlikar, M. and Ramachandran, G.: Inverse Methods for Analysing Aerosol Spectrometer Measurements: A Critical Review, Journal of Aerosol Science, 30, 413-437, https://doi.org/10.1016/S0021-8502(98)00066-4, 1999.

Knutson, E. O. and Whitby, K. T.: Aerosol Classification by Electric Mobility: Apparatus, Theory, and Applications, Journal of Aerosol Science, 6, 443-451, https://doi.org/10.1016/0021-8502(75)90060-9, 1975.

Krakauer, N. Y., Schneider, T., Randerson, J. T., and Olsen, S. C.: Using Generalized Cross-Validation to Select Parameters in Inversions for Regional Carbon Fluxes, Geophysical Research Letters, 31, https://doi.org/10.1029/2004GL020323, 2004.

Kreidenweis, S. M., Petters, M., and Lohmann, U.: 100 Years of Progress in Cloud Physics, Aerosols, and Aerosol Chemistry Research, Meteorological Monographs, 59, 11.1-11.72, https://doi.org/10.1175/AMSMONOGRAPHS-D-18-0024.1, 2019.

Kuang, C.: Scanning Mobility Particle Spectrometer Instrument Handbook, Tech. Rep. DOE/SC-ARM-TR-147, U.S. Department of Energy, Office of Science, ARM Climate Research Facility, 2016.

Lampe, J., Reichel, L., and Voss, H.: Large-Scale Tikhonov Regularization via Reduction by Orthogonal Projection, Special Issue dedicated to Danny Sorensen's 65th birthday, 436, 2845-2865, https://doi.org/10.1016/j.laa.2011.07.019, 2012.

Lira, M., Iyer, R., Trindade, A. A., and Howle, V.: QR Versus Cholesky: A Probabilistic Analysis, International Journal of Numerical Analysis and Modeling, 1, 114-121, 2016. 
https://doi.org/10.5194/amt-2021-51

Preprint. Discussion started: 17 March 2021

(C) Author(s) 2021. CC BY 4.0 License.
Atmospheric

Measurement

Techniques

Discussions

Lopez-Yglesias, X. F., Yeung, M. C., Dey, S. E., Brechtel, F. J., and Chan, C. K.: Performance Evaluation of the Brechtel Mfg. Humidified Tandem Differential Mobility Analyzer (BMI HTDMA) for Studying Hygroscopic Properties of Aerosol Particles, Aerosol Science and Technology, 48, 969-980, https://doi.org/10.1080/02786826.2014.952366, 2014.

Mahish, M., Jefferson, A., and Collins, R. D.: Influence of Common Assumptions Regarding Aerosol Composition and Mixing State on Predicted CCN Concentration, Atmosphere, 9, https://doi.org/10.3390/atmos9020054, 2018.

Marinescu, P. J., Levin, E. J. T., Collins, D., Kreidenweis, S. M., and van den Heever, S. C.: Quantifying Aerosol Size Distributions and Their Temporal Variability in the Southern Great Plains, USA, Atmos. Chem. Phys., 19, 11 985-12 006, https://doi.org/10.5194/acp-1911985-2019, 2019.

Martin, A. C., Cornwell, G. C., Atwood, S. A., Moore, K. A., Rothfuss, N. E., Taylor, H., DeMott, P. J., Kreidenweis, S. M., Petters, M. D., and Prather, K. A.: Transport of Pollution to a Remote Coastal Site during Gap Flow from California's Interior: Impacts on Aerosol Composition, Clouds, and Radiative Balance, Atmos. Chem. Phys., 17, 1491-1509, https://doi.org/10.5194/acp-17-1491-2017, 2017.

Mikhailov, E., Vlasenko, S., Niessner, R., and Pöschl, U.: Interaction of Aerosol Particles Composed of Protein and Saltswith Water Vapor: Hygroscopic Growth and Microstructural Rearrangement, Atmospheric Chemistry and Physics, 4, 323-350, https://doi.org/10.5194/acp4-323-2004, 2004.

Mogensen, P. K. and Riseth, A. N.: Optim: A Mathematical Optimization Package for Julia, Journal of Open Source Software, 3, 615, https://doi.org/10.21105/joss.00615, 2018.

Müller, D., Chemyakin, E., Kolgotin, A., Ferrare, R. A., Hostetler, C. A., and Romanov, A.: Automated, Unsupervised Inversion of Multiwavelength Lidar Data with TiARA: Assessment of Retrieval Performance of Microphysical Parameters Using Simulated Data, Applied Optics, 58, 4981-5008, https://doi.org/10.1364/AO.58.004981, 2019.

Oxford, C. R., Dang, A. J., Rapp, C. M., and Williams, B. J.: Interpretation of Volatility Tandem Differential Mobility Analyzer (V-TDMA) Data for Accurate Vapor Pressure and Enthalpy Measurement: Operational Considerations, Multiple Charging, and Introduction to a New Analysis Program (TAO), Aerosol Science and Technology, 54, 410-425, https://doi.org/10.1080/02786826.2019.1709617, 2020.

Park, K., Dutcher, D., Emery, M., Pagels, J., Sakurai, H., Scheckman, J., Qian, S., Stolzenburg, M. R., Wang, X., Yang, J., and McMurry, P. H.: Tandem Measurements of Aerosol Properties-A Review of Mobility Techniques with Extensions, Aerosol Science and Technology, 42, 801-816, https://doi.org/10.1080/02786820802339561, 2008.

Petters, M. D.: A Language to Simplify Computation of Differential Mobility Analyzer Response Functions, Aerosol Science and Technology, 52, 1437-1451, https://doi.org/10.1080/02786826.2018.1530724, 2018.

Phillips, D. L.: A Technique for the Numerical Solution of Certain Integral Equations of the First Kind, Journal of the ACM, 9, 84-97, https://doi.org/10.1145/321105.321114, 1962.

Rader, D. and McMurry, P.: Application of the Tandem Differential Mobility Analyzer to Studies of Droplet Growth or Evaporation, Journal of Aerosol Science, 17, 771-787, https://doi.org/10.1016/0021-8502(86)90031-5, 1986.

Rawat, V. K., Buckley, D. T., Kimoto, S., Lee, M.-H., Fukushima, N., and Hogan, C. J.: Two Dimensional Size-Mass Distribution Function Inversion from Differential Mobility Analyzer-Aerosol Particle Mass Analyzer (DMA-APM) Measurements, Journal of Aerosol Science, 92, 70-82, https://doi.org/10.1016/j.jaerosci.2015.11.001, 2016.

Reineking, A. and Porstendörfer, J.: Measurements of Particle Loss Functions in a Differential Mobility Analyzer (TSI, Model 3071) for Different Flow Rates, Aerosol Science and Technology, 5, 483-486, https://doi.org/10.1080/02786828608959112, 1986.

Riemer, N., Ault, A. P., West, M., Craig, R. L., and Curtis, J. H.: Aerosol Mixing State: Measurements, Modeling, and Impacts, Reviews of 
https://doi.org/10.5194/amt-2021-51

Preprint. Discussion started: 17 March 2021

(c) Author(s) 2021. CC BY 4.0 License.

(c) (i)

Atmospheric

Measurement

Techniques

Discussions

Royalty, T. M., Phillips, B. N., Dawson, K. W., Reed, R., Meskhidze, N., and Petters, M. D.: Aerosol Properties Observed in the Subtropical North Pacific Boundary Layer, Journal of Geophysical Research: Atmospheres, 122, 9990-10,012, https://doi.org/10.1002/2017JD026897, 2017.

Russell, L. M., Zhang, S.-H., Flagan, R. C., Seinfeld, J. H., Stolzenburg, M. R., and Caldow, R.: Radially Classified Aerosol

Detector for Aircraft-Based Submicron Aerosol Measurements, Journal of Atmospheric and Oceanic Technology, 13, 598-609, https://doi.org/10.1175/1520-0426(1996)013<0598:RCADFA>2.0.CO;2, 1996.

Shen, C., Zhao, G., and Zhao, C.: Effects of Multi-Charge on Aerosol Hygroscopicity Measurement by a HTDMA, Atmospheric Measurement Techniques, 14, 1293-1301, https://doi.org/10.5194/amt-14-1293-2021, 2021.

Shingler, T., Sorooshian, A., Ortega, A., Crosbie, E., Wonaschütz, A., Perring, A. E., Beyersdorf, A., Ziemba, L., Jimenez, J. L., Campuzano-Jost, P., Mikoviny, T., Wisthaler, A., and Russell, L. M.: Ambient Observations of Hygroscopic Growth Factor and f(RH) below 1: Case Studies from Surface and Airborne Measurements, Journal of Geophysical Research: Atmospheres, 121, 13,661-13,677, https://doi.org/10.1002/2016JD025471, 2016.

Sipkens, T., Olfert, J., and Rogak, S.: Inversion Methods to Determine Two-Dimensional Aerosol Mass-Mobility Distributions: A Critical Comparison of Established Methods, Journal of Aerosol Science, 140, 105 484, https://doi.org/10.1016/j.jaerosci.2019.105484, 2020.

Stolzenburg, M. and McMurry, P. H.: TDMAfit User's Manual, Tech. Rep. Technical Report, PTL Publication No. 653, University of Minnesota, Department of Mechanical Engineering, Particle Technology Laboratory, 1988.

Stolzenburg, M. R. and McMurry, P. H.: Equations Governing Single and Tandem DMA Configurations and a New Lognormal Approximation to the Transfer Function, Aerosol Science and Technology, 42, 421-432, https://doi.org/10.1080/02786820802157823, 2008.

Suda, S. R. and Petters, M. D.: Accurate Determination of Aerosol Activity Coefficients at Relative Humidities up to $99 \%$ Using the Hygroscopicity Tandem Differential Mobility Analyzer Technique, Aerosol Science and Technology, 47, 991-1000, https://doi.org/10.1080/02786826.2013.807906, 2013.

Swietlicki, E., Hansson, H. C., Hämeri, K., Svenningsson, B., Massling, A., Mcfiggans, G., Mcmurry, P. H., Petäjä, T., Tunved, P., Gysel, M., Topping, D., Weingartner, E., Baltensperger, U., Rissler, J., Wiedensohler, A., and Kulmala, M.: Hygroscopic Properties of Submicrometer Atmospheric Aerosol Particles Measured with H-TDMA Instruments in Various Environments-a Review, Tellus B: Chemical and Physical Meteorology, 60, 432-469, https://doi.org/10.1111/j.1600-0889.2008.00350.x, 2008.

Talukdar, S. S. and Swihart, M. T.: An Improved Data Inversion Program for Obtaining Aerosol Size Distributions from Scanning Differential Mobility Analyzer Data, Aerosol Science and Technology, 37, 145-161, https://doi.org/10.1080/02786820300952, 2003.

Tikhonov, A. N.: Solution of Incorrectly Formulated Problems and the Regularization Method, Soviet Mathematics Doklady, 4, 1035-1038, 1963.

Twomey, S.: On the Numerical Solution of Fredholm Integral Equations of the First Kind by the Inversion of the Linear System Produced by Quadrature, J. ACM, 10, 97-101, 1963.

Uin, J. and Smith, S.: Southern Great Plains (SGP) Aerosol Observing System (AOS) Instrument Handbook, Tech. Rep. DOE/SC-ARM-TR267, U.S. Department of Energy, Office of Science, ARM Climate Research Facility, 2020.

Wang, S. C. and Flagan, R. C.: Scanning Electrical Mobility Spectrometer, Aerosol Science and Technology, 13, 230-240, https://doi.org/10.1080/02786829008959441, 1990.

Wiedensohler, A.: An Approximation of the Bipolar Charge Distribution for Particles in the Submicron Size Range, Journal of Aerosol Science, 19, 387-389, https://doi.org/10.1016/0021-8502(88)90278-9, 1988. 
https://doi.org/10.5194/amt-2021-51

Preprint. Discussion started: 17 March 2021

(c) Author(s) 2021. CC BY 4.0 License.

(c) (1)

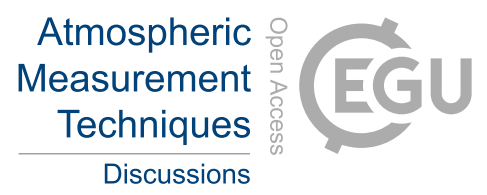

Wolfenbarger, K. J. and Seinfeld, J. H.: Inversion of Aerosol Size Distribution Data, Journal of Aerosol Science, 21, 227-247, https://doi.org/10.1016/0021-8502(90)90007-K, 1990.

700 Wu, Z., Birmili, W., Poulain, L., Wang, Z., Merkel, M., Fahlbusch, B., van Pinxteren, D., Herrmann, H., and Wiedensohler, A.: Particle Hygroscopicity during Atmospheric New Particle Formation Events: Implications for the Chemical Species Contributing to Particle Growth, Atmos. Chem. Phys., 13, 6637-6646, https://doi.org/10.5194/acp-13-6637-2013, 2013.

Zhang, S.-H., Akutsu, Y., Russell, L. M., Flagan, R. C., and Seinfeld, J. H.: Radial Differential Mobility Analyzer, Aerosol Science and Technology, 23, 357-372, https://doi.org/10.1080/02786829508965320, 1995. 